$$
N 63-20245
$$

\title{
INVESTIGATION OF PROBLEMS IN THERMAL CONVECTION
}

\author{
J. R. Herring \\ Goddard Institute for space studies \\ National Aeronautics and space Administration \\ New York 27, N.Y.
}

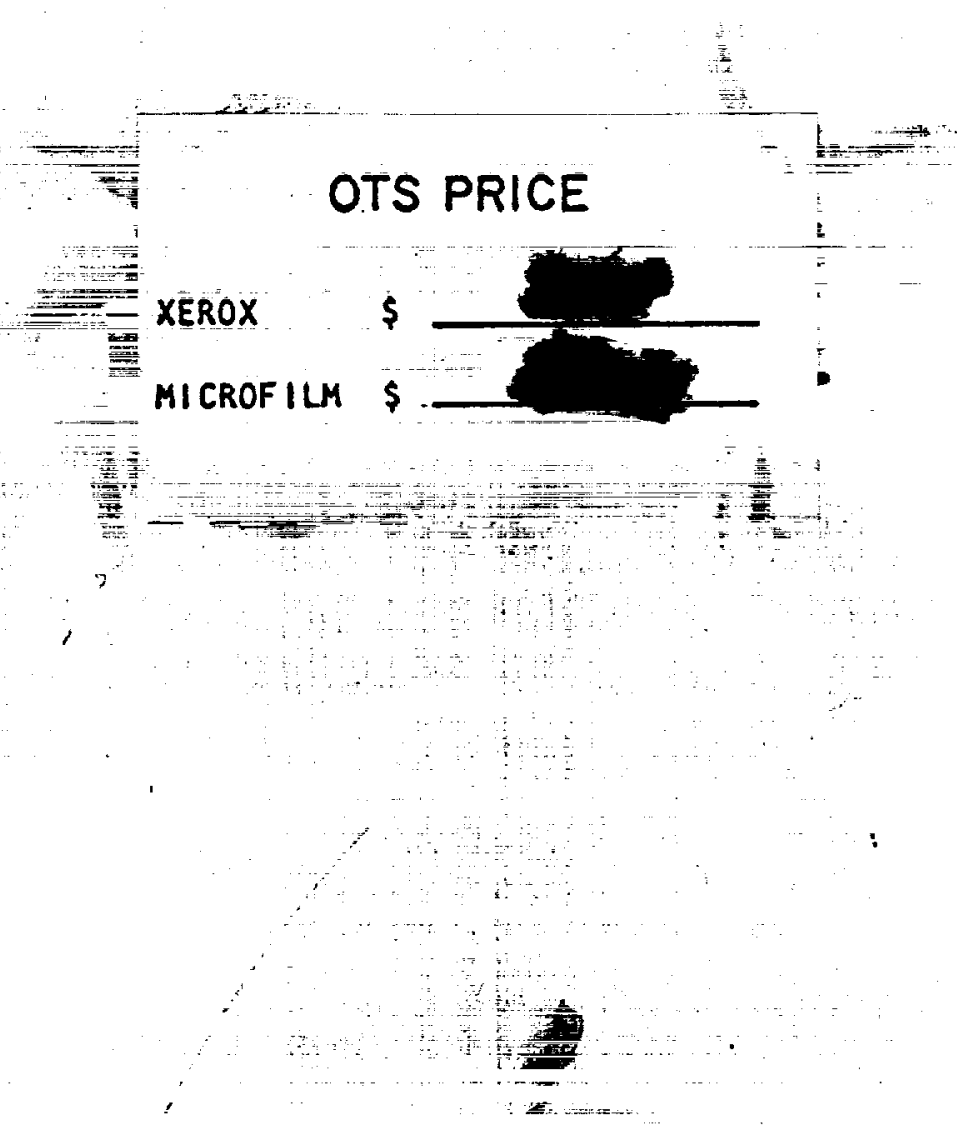


ABSTRACT

The thermal convection equations for a thin layer of fluid are solved numerically as an initial value problem. The calculations include only those nonlinear terms which have the form of an interaction of a fluctuation in the velocity and temperature with the mean temperature fieid. In the present calculations, the velocity and temperature fluctuations have one horizontal wave number, and satisfy free boundary cunditions on two conducting horizontal surfaces.

The computed steady state veiocity and cemperature amplitudes show many of the observed qualitative reatu: es. In particular, the experimentally observec sundary iayering, of the mean temperature field is correctly prosides. and, ac large Rayleigh number, the total heat transport . EO Eno to de proportional to the cube root of the Rayleigh number wovided the fluctuating temperature and velocity amp:-icucias have thai horizontal wave number which maximizes the tot: nes: transpo: However, the heat transport found here for ree ocuradaries is absut three times the experimental value for $\cdots, \cdots$ boundaries. Th: mean temperature gradient can become $A, a$. i near the boundaries for large Rayleigh numbers anc sit horit-ntal scale motions. 



\section{Introduction}

This paper describes the resulis of a numerical investigation of the thermal convection equations for a thin layer of fluid confined between two plates on which free boundary conditions are employed. Our theoretical procedure is to include only those nonlinear terms which describe the interaction of the mean temperature with velocity and temperature fluctuations. That is, those terms responsible for eddy viscosity and eddy conductivity effects on the turbulence itself are omitted. The above eddy terms (hereafter referred to as fluctuating selfinteractions) are discarded in a physically consistent manner, so that no unrealistic behavior results from their omission.

The motivation for this "research is to discover quantitatively to what extent the turbulent convection problem can be comprehended without the fluctuating self-interactions. The system of equations obtained by deleting these terms corresponds to closing the hierarchy of moment equations at the first nontrivial level by discarding third order cumulants. The resulting system of equations is complete and involves no empirical parameters. Moreover, the gross energetics of the flow are preserved.

The method of numerical solution consists in integrating the Fourier amplitudes of the velocity and temperature fields forward in time until the steady state is achieved. This procedure has the advantage of assuring the stability of the final state provided a sufficient range of initial data is sampled. The present 
calculations, carried out on the IBM 7090, contain one horizontal wave number and enough vertical wave numbers to ensure the elimination of truncation errors. In the numerical analysis, we have set the Prandt $I^{\prime}$ nunber equal to unity. However, inspection of the equations which omit the fluctuating self-interactions shows that the heat transport is not a function of Prandtl number, if the system is steady. ${ }^{1}$

The calculated velocity and temperature fields show many of the qualitative features of the experimentally determined fields. In particular, at large Rayleigh number, $R$, the total heat transport is found to se proportional to $\mathrm{R}^{1 / 3}$, provided the velocity and temperature fluctua:ion fields have that horizontal wave number which maximizes the heat transport. However, the heat transport found here for free-boundary conditions is three times the experimental value for rigid-boundary conditions. Preliminary numerical studies of the rigid-boundary problem indicate that for large Rayleigh numbers $\left(R \sim 10^{6}\right)$ the heat transport is about a factor of 2.3 smaller than that for free-boundaries and therefore approximately 50 percent higher than the experiment. Thus, it appears that the boundary conditions are quite important in producing the experimental heat transport. The system has two additional failings: it turns out that the fluctuating amplitudes are steady in time and the horizontal plan form of the motions is indeterminate.

The mean temperature gradient at low $\mathrm{R}$ closely resembles the experimental temperature profiles. At large Rayleigh numbers $\left(R \sim 10^{6}\right)$, the gross features of the temperature profiles are correctly predicted by our system. The computed mean temperature gradients are large in a thin layer adjacent to boundary and are 
quite small in the body of the fluid. The gradients near the boundary can become negative for motions of large horizontal scale, but remain positive for motions of a sufficiently smajl. horlzontal scale.

The stability of the steady state solutions against infinitesimal perturbation at other horizontal wave numbers is also investigated and the regions of instability are delineateci: These results closely parallel perturbation results at low Rayleigh number and support the idea that the most stable solutior: is near the one transporting the most heat flux (Malkus and Veronis, 1958). A finite amplitude stability study, and the associatced development of a several-horizontal-wave-number system co stendy state will be the topic of a future investigation.

The idea that the important features of the conve ation problem are contained in the system which onits the fluctuating self-interactions is implicit in the theory of convection advanced by Malkus (1954). In the original formulation of his theory. Malkus sought a maximum for the heat transport subject to the constraint that the temperature gradient be positive, and that there be a smallest scale of motion participating in the advective heat transport. The smallest scale is supposed to be determined by the requirement that it be marginally stable in the presence of the mean temperature gradient occuring in the fluid. The smallest scale so determined Eurnished a cut off in the cosine representation of the mean temperatu:e gradient. The assumption that the heat transport was maximum under the above constraint:s then led to an explicit form for the temperature gradient. 
A more recent formulation of the Malkus theory by Splegel (1962) replaces the cosine representation of the temperature gradient by an expansion in terms of the set of eigenfunctions, which are marginally stable on the mean temperature gradient. This version of the Malkus theory is exactly equivalent to the system considered here, provided the horizontal scale of the motions is such that the mean temperature gradient is everywhere positive. In this sense, our numerical results contain, as a " special case, the exact solutions to the Malkus theory for one horizontal wave number and free boundaries.

In this connection, a comparison of our computed temperature gradients with the predictions of the Malkus theory is relevant. In making this comparison, we must keep in mind that the systom considered here is explicitly confined to only one horizontal wave number, whereas Malkus makes ro explicit references to the nature of the horizontal-wave-number spectrum. We do not confixm the $\mathrm{z}^{-2}$ law for the gradient outside the boundary layer as predicted by Malkus, nor do we find a sharp cut off in the cosine rpectrum of the temperature gradients.

At low Rayleigh numbers $(R<2000)$ our numerical results are in agreement with the calculations of Malkus and veroni:A (1958) and Kuo (1961), who have obtained perturbation solutions to the convection equations. A procedure similar to ours has been used by saltzman (1961) in studying the complete convection equations for $R \lessgtr 6000$. Our approach differs from his in that we are able to allow very many vertical modes to be excited, 
whe:eas his results are limited to one vertical mode. Our results indicate that it is essential to allow many more vertical modes than horizontal modes, if large truncation errors are to be avoided. Thus, at $R=4000,10$ vertical modes must be included to obtain realistic temperature profiles.

\section{Theory}

a) The Equation of Motion and Boundary Conditions We consider a thin layer of fluid confined between two infinitely conducting plates located at $z=0$ and $z=d$. The lower plate is maintained at zero degrees, and the top plate at a temperature $-T_{o^{\prime}}$ on an arbitrary temperature scale. The direction of gravity is specified by the unit vector $\hat{-k}$. The equations appropriate for our system are the Boussinesq approximations ${ }^{2}$ to the Navier-stokes equations. We shall write these equations in a form in which the velocity and temperature $(\vec{v}$ and $T)$ as well as the coordinate and time $(\vec{r}$ and $t)$ are nondimensional. The only parameters entering the equations are then the Rayleigh number, $R$, and the Prandt $l$ number, $\pi$. The equations are

$$
\begin{gathered}
7 \cdot \vec{v}=0 \\
\left(\frac{1}{\pi} \frac{\partial}{\partial t}-\nabla^{2}\right) \nabla^{2} \vec{v}=\frac{1}{\sigma} \nabla_{x}(7 \times(\vec{v} \cdot 7 \vec{v})+R 7 x(7 \times \hat{k T}) \\
\left(\frac{\partial}{\partial t}-\nabla^{2}\right) \mathrm{T}=-\nabla \cdot(\overrightarrow{\mathrm{v} T})
\end{gathered}
$$


Equation (2) is actually the double curl of the momentum equation, and lience the pressure variable is absent. The nondimensional variables are related to the dimensional ones (denoted by primes) in the following way:

$$
\begin{aligned}
& \vec{v}=\frac{d}{x} \vec{v}^{\prime}, \\
& T=T^{\prime} / d \\
& \vec{r}=\vec{r}^{\prime} / d \\
& t=\frac{x}{d^{2}} t^{\prime}
\end{aligned}
$$

Here $x$ is the thermometric diffusivity of the fluid.

The boundary conditions on the velocity field are derived from the requirement that the fluid exert no shear on the confining plates. This, together with the continuity equation, implies that all even derivatives of the vertical velocity, w, vanish on the boundary. In the nondimensional notation the boundary conditions are,

$$
\frac{\partial^{2 m}}{\partial z^{2 m}} w(0, t)=\frac{\partial^{2 m}}{\partial z^{2 m}} w(1, t)=0, m=0,1,2, \ldots
$$

and

$$
T(0, t)=0 ; \quad T(1, t)=-1 .
$$

b) Discard of the Fluctuating Self-Interactions

It is convenient to resolve the temperature field into a horizontal mean plus a fluctuating part; 


$$
T=-z+\psi(z, t)+\Theta(\vec{r}, t)
$$

Here, $\psi(z, t)$ is a horizontally averaged distortion or the conduction state and $\Theta(\vec{r}, t)$ is the fluctuation of the temperature from its distorted value. In view of the boundary condition (5), and the interpretation of $\Theta$ as a fluctuation from the horizontal mean, we. mày write.

$$
\begin{aligned}
& \Downarrow(0, t)=\psi(1, t)=0 \\
& \uplus(x, y, 0, t)=\uplus(x, y, 1, t)=\bar{M}=0
\end{aligned}
$$

The ban on equation (8) indicates an average over the horizontal. We now "introduce equation ( 6 ) into equations (1), (2) and ( 3 ) and subtract from each of the resulting equations their respective horizontal mean. We find

$$
\begin{aligned}
& \left(\frac{1}{\sigma} \frac{\partial}{\partial t}-\nabla^{2}\right) \nabla^{2} w=R \nabla_{1}{ }^{2} \Theta+\frac{1}{\sigma}\{\nabla \times \nabla \times(\vec{v} \cdot \nabla \vec{v})\}_{2} \\
& \left(\frac{\partial}{\partial t}-\nabla^{2}\right) \Theta=\left(1-\frac{\partial \psi}{\partial z}\right) w-\nabla \cdot(v \Theta-k \overline{w \Theta}) \\
& \left(\frac{\partial}{\partial t}-\frac{\partial^{2}}{\partial z^{2}}\right) \psi=-\frac{\partial}{\partial z} \overline{w \Theta}
\end{aligned}
$$

There are two more equations, for the $\mathrm{x}$ and $\mathrm{y}$ components of the velocity field, but these are not necessary for our problem. The last terms in the equations above for,$W$ and $\oplus$ have the form of a deviation of a bilinear fluctuating quantity from its horizontal mein (fluctuating self-interaction). By discarding these terms we obtain the system to be investigated; 


$$
\left(\frac{1}{\sigma} \frac{\lambda}{\lambda t}-\nabla^{3}\right) \nabla^{a} W=R \nabla_{1}{ }^{2} \Theta
$$

$$
\begin{gathered}
\left(\frac{\partial}{\partial t}-\nabla^{a}\right) \Theta=\beta w \\
\vdots \\
\left(\frac{\partial}{\partial t}-\frac{\partial^{2}}{\partial z^{2}}\right) \psi=-\frac{\partial}{\partial z} \overline{w \Theta}
\end{gathered}
$$

where: $\quad \beta(z)=1-\frac{\partial \psi}{\partial z}=-\frac{\partial}{\partial z} \bar{T}$

The significance of omitting fluctuating self-interaction can be expressed formally by examining the hierarchy of moment edultions obtained from equations (1)-(3). By multiplying equations (2) and (3) by $v\left(t^{\prime}\right)$ and $T\left(t^{\prime}\right)$ and ensemble-averaging the appropriate suins of the resulting equations, we obtain the time evolution equations for the correlation coefficients $\left\langle\mathrm{v}_{i} \mathrm{v}_{j}{ }^{\prime}\right\rangle$, and $\left\langle\mathrm{v}_{i} \mathrm{~T}^{\prime}\right\rangle$, and $\left\langle\mathrm{T}^{\prime} \mathrm{T}^{\prime}\right\rangle$. These equations couple the above second order moments to the transfer terms, which are cubic in $\vec{v}$ and $T$.

Since the system contains a non-vanishing first-order moment, $\forall$, the transfer terms contain both correlated third-order moments (cumulants) and products of first order moments with second-order moments. The discarding of the fluctuating self-interaction. then corresponds to closing the system of moment equations by discarding the third order cumulants. ${ }^{3}$ In the absence of mean fields this procedure would be empty.

We must now verify that our procedure of deleting third-ordcr cumulants does not lead to physically unrealistic results. For our procedure to be acceptable, the system of equations (9), (10) and (11) 
must obey the conservation laws associated with the complete set of convection equations, and they must be free from unphysical consequences of the sort recently discussed by Ogura (1962). for a similar problem in isotropic turbulence. Ogura has demonstrated that the assumption of zero forth-order cumulants and nonzero third-order cumulants is incompatible with a positive energy spectrum for all wave numbers. 4

With regard to the last point, it should be noted that the positive definite character of the kinetic energy wave number spectrum and the spectrum for the square of the temperature field follows : directly from the fact that it is possible to write the equations which delete third order cumulants in terms of amplitudes rather than moments. We observe that the amplitude equations (9), (10) and (11) all have real coefficients; hence, the square of any amplitude will remain positive for all time if the amplitude is initially a real number.

The conservation of entropy and kinetic energy are also preserved without the fluctuating self-interactions. By multiplying equation (10) by $\Theta$, equation (11) by $\psi$, and adding, we obtain after integrating over the entire volume of the fluid, the equation of conservation of entropy,

$$
\frac{1}{2} \frac{\partial}{\partial t}\left\{|\Theta|+|\psi|^{2}\right\}_{v}+\left\{|\nabla \psi|^{2}+|\nabla \Theta|^{2}\right\}_{v}=\{\omega \Theta\}_{v} .
$$


Here the $v$ subscript indicates an intagration over the entire volune of thi system.

We observe that equation (12), with a corresponding one for the conservation of the kinetic energy ${ }^{5}$ of the flow are exactly the same as those with the fluctuating self-interaction included. Contributions from the latter may be reduced to surface integrals which vanish.

\section{C) Fouxier Decomposition of the Equations}

It is convenient to work with the Fourier components of the equations (9)-(11) rather than their space-variable form. The free boundary conditions make the sine series appropriate. We therefore write:

$$
\begin{aligned}
& w(r, t)=\sum_{n, \alpha} f_{\alpha}(x, y) w_{n}^{\alpha} \sin n \pi z \\
& \Theta(\vec{r}, t)=\sum_{n, \alpha} f_{\alpha}(x, y) \Theta_{n}^{\alpha} \sin n \pi z \\
& \psi(z, t)=\sum_{n} \psi_{n} \sin n \pi z
\end{aligned}
$$

Here $f_{\alpha}(x, y)$ is ain arbitrary set of orthonormal functions generated by the operator $7_{1}{ }^{2}$, and obeying appropriate periodic boundary conditions in the horizontal:

$$
7_{1}{ }^{2} f_{\alpha}(x, y)=-\pi^{2} \alpha^{2} f_{\alpha}(x, y)
$$


$-11-$

and

$$
\left|\overline{\mathrm{f}_{\alpha} \mathrm{f}_{\alpha}{ }^{\prime}}\right|^{2}=\delta_{\alpha \alpha^{\prime}}
$$

Introducing the above representation into equations (9), (10), and (11) gives the following set of equations for the amplitudes $w_{n}, \Theta_{n}$, and $\psi_{n}$ :

$$
\begin{aligned}
& \left(\frac{1}{\sigma} \frac{\partial}{\partial \tau}+n^{2}+\alpha^{3}\right) w_{n}^{\alpha}=\frac{\lambda \alpha^{2}}{n^{2}+\alpha^{2}}{ }_{n}^{\alpha}{ }^{\alpha} \\
& \left(\frac{\partial}{\partial \tau}+n^{a}+\alpha^{a}\right) \Theta_{n}^{\alpha}=\omega_{n}^{\alpha}-\frac{\pi}{2} \sum_{p=1} \cdot p^{\psi} p\left(\omega_{n+p}^{\alpha}+\sigma(n-p) \omega^{\alpha}|n-p|\right) \\
& \left(\frac{\partial}{\partial \tau}+n^{2}\right) \Psi_{n}=\frac{\pi n}{2} \sum_{p=1} \sum_{\alpha} \omega_{p}^{\alpha}\left(\left(^{\alpha}{ }_{n+p}+\sigma(p-n) \Theta^{\alpha}|n-p|\right)\right.
\end{aligned}
$$

where

$$
\begin{aligned}
& \lambda=R / \pi^{4}, \\
& \tau=\pi^{2} t \\
& \omega_{n}=\omega_{n} / \pi^{2}
\end{aligned}
$$

and

$$
\begin{aligned}
\sigma(x) & =1, x>0 \\
& =0, x=0 \\
& =-1, x<0 .
\end{aligned}
$$


Manipulation of the convolution terms in (14) and (15) is aided by the following identities:

$$
\sum_{p} A_{p}\left(B_{n+p}+\sigma(n-p) B|n-p|\right)=\sum_{p} B_{p}\left(A_{|n-p|}-A_{n+p}\right),
$$

and,

$$
\sum_{p} A_{p}\left(B_{n+p}+\sigma(p-n) B_{|p-n|}\right)=\sum_{p} B_{p}\left(A_{n+p}+\sigma(p-n) A_{|p-n|}\right) .
$$

There are two conservation equations derivable from (14) and (15). The first is the Fourier repreientation of equation (12) for conservation of entropy. The other is the equation that partitions the total heat flux between conduction and convection; and it is derived by multiplying equation (15) by $1 / \mathrm{n}$ and summing over $\mathrm{n}$. We find

$$
\sum_{n}\left(\frac{1}{n^{2}} \frac{\partial}{\partial t}+1\right) \beta_{n}=\frac{\pi^{2}}{2} \sum_{n, \alpha} \omega_{n}^{\alpha} e_{n}^{\alpha}
$$

where

$$
B_{n}=-\pi_{n} \psi_{n} .
$$

Here the $\beta_{n}^{\prime} s$ are the cosine transform of the mean temperature gradient. In the statistically steady state, equation (16) is the equation for the total heat flux, which is a constant of motion for the system. We now introduce a quantity $N(t)$, the total heat flux at the lower boundary: 


$$
N(t)=1+\sum_{1}^{\infty} B_{n}(t)
$$

If the mean amplitudes are constant,

$$
N=1+\frac{\pi^{2}}{2} \sum_{n, \alpha} \omega_{n}^{\alpha} \Theta_{n}^{\alpha}
$$

In our units, the conduction state transports unit heat flux and this equation is the nondimensional form for the famillar equation for the total heat flux.

\section{d. Structure of the Equation}

Before proceeding to the numerical results, we give a bricl resume of the pertinent qualitative features. of the system defined by equations (13), (14) and (15). First of all, we note that the horizontal wave numbers, $\alpha$, are coupled only in their effect on the mean temperature field $\psi$. This interaction occurs diagonally in the sense that each $\alpha$-interacts only with itself. As a consequence there is a degeneracy in the horizontal plan form of the motion; the system is insensitive to the particular cell shape. Moreover, the number of $\alpha^{\prime} s$ is also indeterminate. The simplest situation is to have a single $\alpha$ support the motion and we investigate only this case here.

A single $\alpha$ will give nontrivial answers for the amplitudes $w$ and $(0)$ only if it lies within a certain range. The range of $\alpha$ which will not support convection is obtained by assuming $w$ and 
to be small, and demanding that they subsequently decay. If $w$ and $\circledast$ are small, $\psi$ will be small to second order and our question - is equivalent to that of marginal stability. ${ }^{6}$ The system then will. not support convection if

$$
\left.\frac{\left(1+\alpha^{2}\right.}{\alpha^{2}}\right)^{3} \geq \frac{R}{\pi^{4}}
$$

Conversely, we assume that the steady state values of $w$ and $\Theta$ will be nonzero if a lies in the range complementary to (18).

The time behavior of the system is complicated by nonlinear effects. In the approach to the steady state, our numerical results indicate that the system executes overdamped oscillations with an ever increasing period of oscillation. This last remark is understandable since $w$ and $\Theta$ become marginally stable as $t \rightarrow \infty$.

If the mean field, $\psi$, is statistically steady as $t \rightarrow \infty$, we mey use a theorem of Spiegel ${ }^{7}$ to show that $w$ and $\oplus$ are indepentent of time. Spiegel has shown that the principle of exchange of stability is valid (for free boundaries) in the presence of the mean gitidient corresponding to the steady state solution to the mean temperature field given by (15). This implies that the growth rates for the appropriate eigen-function expansion for $w$ and $\Theta$ must all be zero in order for there to be a statistically steady state.

\section{Numerical Procedure}

In performing the numerical integration, we discard from the onset those Fourier amplitudes which will be zero in the steady state. 
We assume that the steady state amplitudes (", $\Theta$, and 8 have even parity about the mid point $z=1 / 2$. This means that the even sine mocies of $" \prime$ and $\theta$, and the odd cosine modes of 8 will have zero amplitude in the steady state. We therefore put their initial values equal to zero. The equations of motion (13), (14) and (15) then imply that the odd parity moies will remain zero for all subsequent time. Defining $\bar{\beta}_{n}=\beta_{2 n}$, we may rewrite (14) and (15) in a more convenient form:

$$
\begin{aligned}
& \left(\frac{\partial}{\partial \tau}+n^{2}+\sigma^{2}\right) \infty_{n}=u_{n}+\frac{1}{2} \sum_{p=1}^{\infty} \bar{\beta}_{p}\left(\omega_{n+2 p}+\sigma(n-2 p) \omega_{|n-2 p|}\right) \\
& \left(\frac{\partial}{\partial \tau}+4 n^{2}\right) \bar{\beta}_{n}=-2 \pi^{2} n^{2} \sum_{p=1}^{\infty} \omega_{p}\left(\mu_{2 n-p}+\sigma(p-2 n) \mu_{\mid}|2 n-p|\right) .
\end{aligned}
$$

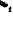
Here, we have dropped the a superscript since we are interested in the system containing only one a. Equation (13) remains unchenged and the total heat flux is computed from equation (17). our procedure for integrating these equations is to assign an initial set of amplitudes to $\omega_{n}, \Theta_{n}, \bar{\beta}_{n}$, and allow the system to evolve to the steady state. In doing so, we must truncate the infinite set of equations. Our procedure in this matter is to set all amplitudes $\omega_{n}, \Theta_{n}, \bar{\varepsilon}_{n}$ of index greater than a certain integer, $n_{0}$, equal to zero. This method of truncation guarantees exact conservation of heat flux and entropy for the abbreviated system. Since $m_{1}$ is generally large and the $\oplus_{n}$ 's decrease rather slowly, we see from (15') that amplitudes for $\vec{\beta}_{n}$ above $n_{n} / 2$ will 
have significant truncation errors. Truncation errors are assumed to be negligible if increasing $n_{0}$ does not appreciably alter the - value of the total heat transport The total number of $\bar{B}_{N}$ modes included in these calculations ranged from 20 modes at $R=4000$ to 80 modes at $R=10^{6}$. The errors in the total heat transport, due to the above are estimated to be less than one part in $10^{3}$

The integration forward in time was continued until constancy of heat flux (16) and entropy (12) was achieved to one part in $10^{1}$. The time, in $T$ units, necessary to achieve this ran from $\sim 1.4$ at $R=4 \times 10^{3}$ to 0.3 at $R=10^{6}$. At high Rayleigh numbers, this criterion was not too satisfactory, since constancy of heat flux and entropy were achieved long before the amplitudes $w$ and if became steady. For these cases, it was necessary to check the time derivatives of the slowest evolving amplitudes, $w_{1}$ and $A_{1}$. The system was observed to be steady if the derivative of $\mathrm{w}_{1}$ was less than 2 percent of $\mathrm{w}_{2}$.

Examples of the time evolution for the total heat transport $N(\tau)$ are given in Figure 1 , for $R=4 \times 10^{3}, 10^{4}, 10^{5}$ and $\alpha=1.5$. The system was started in the conduction state at $\tau=0$, with all fluctuating amplitudes $\omega_{N}$ and $\theta_{N}$ equal to zero, except $\omega_{1}$, which had an initial value of unity. The convection is seen to develop initially by way of large oscillations, and to decay to the steady state with overdamped oscillations, whose period becomes increasingly larger. The time scale of the initial oscillations in these curves is of the order of the growth rate time in the conduction state. 
IV Discussion of Results

The computed steady state amplitudes are shown in figures 2-13. The normalization for $B, w$, and $\Theta$ are given in the captions, while $\bar{T}(z)$ requires no normalization. The graphs of $\bar{T}(z)$ are in a

- reflected coordinate system to conform with an accepted proccdurc. The values of $\alpha$ in figures $2-9$ were chosen so that the heat transport is very near its maximum. We now discuss in some detail the physical features of the steady state amplitudes $\omega, \theta, 1$, and $\bar{T}$.

a) The Mean Temperature $T$, and Mean Gradient $B(z)$ The mean fields, $T$ and $B$ in figures $3,5,7,9,11$ and 13 have an interesting behavior near the boundaries. At low Rayleigh number, these fields closely resemble the perturbation results of Malkus and Veronis (1958), but the temperature gradient is slightly negative in the central region. As the Rayleigh number is increased, the negative temperature region collects closer to the boundary while in the central region, the temperature gradient becomes extremely small but positive.

The negative temperature gradient boundary region is apparently produced by an overshoot pheonomenon. These occur typically for motions of large horizontal scale (small $\alpha$ ) and disappear for motion of small horizontal scale. (See figures 9, II and 13.) If the motion has a large horizontal scale, an element of fluid close to the lower plate moves in a region of high temperature for a relatively long time. When it eventually. turns upward, it moves 
unchecked by eddy processes and penetrates the body of the fluid with an excessive heat flux. The mean gradient accommodates this motion by turning negative. The negative $a$ region then checks the velocity field, so that the advective heat transport decreascs towerd the middle of the fluid. We see here evidence for the non-local property of the flow; if the Rayleigh criteria for convection were applicable locally, a negative \& region would not persist in the steady state.

For motions of small h. rizontal. wale (figures 11 and 13) the sltuation is somewhat diferent. In this case, an element: absocbs little heat from th. Tower boundary region and loses it: quickly by conduction because it belongs to a vertically elongated cell pattern. It also loses mentum by viscous drag, and attains its terminal velocity before reaching the central region of the fluid (see figures 10 and 12). To maintain constancy of heat flux the central region must conduct rathor strongly, so that the mean gradient becomes large there.

\section{b) Velocity and Temperature Fluctuations}

The velocity and temperature fluctuation fields are shown in figures $2,4,6,8,10$ and 12 . We observe that the velocity fields, for all Rayleigh numbers, have an extremely large first mode. For example, at $R=4000$, (figure 2) $w_{1}$ represents $99 \%$ of the total velocity amplitude, while at $R=10^{6}$ (figure 8 ) $\mathrm{w}_{1}$ is $95 \%$ of the total. On the other hand, the $\otimes_{\mathrm{n}}$ modes decrease rather. 
slowly as $\mathrm{n}$ increases.

The above behavior of the $\omega_{n}$ and $\Theta_{n}$ spectra displays the character of the nonlinear coupling in our system. Thus, the Lerm $\omega_{1} \otimes_{2 n-1}$ tend to be the dominant contributor to $\bar{B}_{n}$ [see equation (15')] for reasonably small $n$. Conversely, terms of the form $\bar{\beta}_{n} \omega_{1}$ and $\bar{B}_{n-1}{ }^{10} 1$. tend to be the dominant contributors to $\Theta_{\mathrm{n}}\left[\right.$ equation $\left.\left(14^{\prime}\right)\right]$. The nonlinear coupling scheme in the equations of motion is therefore highly nondiagonal, as opposed to the case of isotropic turbulence. The strong nondiagonal coupling in the system of Fourier nodes is a result of the distortion of the mean temperature profile combined with the pressure and dissipative forces for incompressible flow. The above forces are directly responsible for the occurrence of sixth-order derivatives in the marginal stability problem, of which the steady state amplitudes $w$ and $\Theta$ are solutions in the presence of the mean field $\beta$. If we solve for the velocit: "ydes $w_{n}$, in the presence of the mean gradient $\beta$, by using the iteration technique of section $v$, we see that the higher modes of $\omega_{n}$ are suppressed by a factor $\sim n^{-6}$. For a reasonable $\beta$; this factor results in the higher $w_{n}$ modes making only a small contribution to $w$.

\section{c) Temperature Gradient Spectrum}

The cosine spectrum of the mean temperature gradient, $\beta$, is given in figure 14 for $R=10^{4}, 10^{5}, 10^{6}$ and $\alpha=1.5$. We have connected the points with a smooth curve for the sake of clarity. 
We notice a tendency for the lower modes to saturate at ${ }^{B_{n}}=2$, which corresponds to the small gradient outside the boundary laycr. In fact, if $R_{n}=2$ for all $n, s(z)$ is a $\delta$ function, and the graident vanishes everywhere except at the boundary, where it becomes stingular. At large Rayleigh numbers, the $A_{n}$ spectrum is nearly Gaussian for small $\mathrm{n}$, but decreases more rapidly at large $\mathrm{n}$.

The tendency for the $\bar{B}_{n}$ 's (for smali $n$ ) to approach 2 as an upper bound is closely connected with the fact that the velocily field is marginally stable on the mean temperature gradient, $P$. This feature is brought out more clearly by examining the relation connecting the mean gradient field, $\bar{R}_{\mathrm{n}}$ and the Rayleigh number $R$. Using the iteration method of Section $V$, we find

$$
\left.\frac{\left(1+\sigma^{2}\right.}{a^{2}}\right)^{3} \frac{\pi^{4}}{R}=1-\frac{1}{2} \bar{\beta}_{1}+\frac{1 / 4}{1-\frac{1}{2} R_{2}} \sum_{1}^{\infty} \frac{\left(1+\alpha^{2}\right)^{3}}{\left[(2 n+1)^{2}+\alpha^{2}\right]^{3}}\left(\bar{B}_{n}-\bar{B}_{n+1}\right) \cdots+\ldots
$$

This series for $R^{-1}$ converges rather rapidly for all the $\bar{R}_{n}$ which have been computed, and the terms explicitly written in equation (19) give $R$ to an accuracy of $\leqslant 20 \%$ at $R=10^{6}$. We note that for this equation to balance at large $R, \bar{R}_{1}$ mus $=$ approach 2 , and the remaining lower modes must decrease rather slowly as $\mathrm{n}$ increases.

The computed spectra (figure 14) are qualitatively quite different from the one derived by Makus. ${ }^{8}$. His spectrum is given by

$$
\bar{\beta}_{n}=2\left(1-\frac{n}{2 n_{0}+1}\right)
$$


Here $2 n_{r}+1$ is a cut-off in the $\bar{p}_{n}$ spectrum, an it is the total heat flux in our unirs. Narginal stability is achieved at a mucls lower Rayleigh number for this spectrum than for the ones computed here.

With regard to the Nalkus theory, figures 10 and 11 are relevant. For this case $\left(R=10^{6}, \alpha=6.0\right)$ the temperature gradient is., everywhere positive except near the boundaries where it approsises zero. The fields in figures 10 and 11 therefore fulfill all the requirements of the Nalkus theory as formulated by spiegel (1962). We note for this case that the total heat flux is $\sim 22$, whereas ralkus obtains a heat transport of $\sim 11$ for free boundaries. In making this comparison, one should remember that these computations were made for a single horizontal wave number, whereas the Valkus presuniably allowed for a full spectrun of a's. However, if we interpret the computed heat transport as an upper bound to the heat transport as the ralkus theory prescribes, we conclude that for free boundaries the actual upper bound is at least a factor of two larger than that obtained by Malkus.

d) The Total Heac Transport as a Function of $\alpha$ and $R$

The total heat transport, as a function of $R$ and $\alpha$ is given in figure 15. The Rayleigh numbers are indicated in the figure. These curves closely resemble the perturbation calculations at. small $\mathrm{R}$, but become increasingly broadened as the Rayleigh number increases. For a given $R$, the heat transport is entirely conductive 
( $N:: 1$ ) if a lies outside the bounds prescribed by equation ( $1 \varepsilon$ ). The value of $\alpha$ wich maximizes the heat transport is $1 / \sqrt{2}$ at tive crit:ical Rayleigh number $(R=657)$, and apparently increases Inearly in $R^{1 / 3}$, at large $R$. It is well represented at high Rayjeigh numbers by the formula:

$$
\sigma_{\max } \simeq 0.7+0.01 \mathrm{R}^{1 / 3}
$$

The data on this point is not entirely conclusive because of the large breadth of the curves. It should be pointed out that equition (20) cannot be a correct asymptotic formula since $a_{\max }$ is froportional to $\mathrm{R}^{1 / 3}$ and the value of $\alpha$ beyond which a single a cannot support convection is proportional to $R^{1 / 4}$ (equation 18 ). 9 An estimate of the Rayleigh number beyond which (20) is incorrect is not warranted by the accuracy of the curves, but according to equations (18) and (20), it is $R \lesssim 10^{18}$.

The maximum heat transport as a function of $R$ is given in figure 16. For $R \geq 3000$ the data is accurately represented by the following $R^{1 / 3}$ law:

$$
\mathrm{N} \cong 0.3 i \mathrm{R}^{1 / 3}
$$

Experimentally, the Nusselt number $N$ is $\sim 0.085 \mathrm{R}^{1 / 3}$, for large $R$, and rigid boundaries (Jakob, 1959). We see no evidence for an intermediate $\mathrm{R}^{1 / 4}$ law, but such a law may only be obtained in the rigid boundary problem. Below $R \sim 10^{3}$, the data fits smoothly lo the perturbation calculation of Malkus and Veronis (1958). 
The discrepancy between (21) and the experiment is parts a result of eddy processes which our prccedure omits. The use of rigid boundary conditions may improve the agreement, but if (21) is corrected for boundary effects as done by Malkus (1960) by decreasing $\mathrm{N}$ by $\left(\frac{657}{1107}\right)^{1 / 3}$, there remains a discrepancy of a factor of 2 . If we choose horizontal wave numbers such that the mean gradicnt is everywhere positive (figures 10 and 11) the discrepancy is reduced to 1.8 . The latter fields, however, have the unattractive feature of having a large temperature gradient in the central region of the fluid.

\section{$V$ Linear Stability of the Fiel Is}

The velocity and temperature fillds we have so far discussed are itable against the introduction of a disturbance of the sarie horizontal wave number for which the fields were computed. This stability is inherent in the method of integrating the equations. The stability of the steady state amplitudes against disturbances at wave numbers $\alpha^{\prime}$ other than that $a$ which supports the convection process has not yet been assured in our calculations. The question of sability of the solutions against disturbances of finite amplitudes leads directly back to the multi-a system of. equations (13), (14) and (15). We should assume a whole spectrurr: of $q$ 's are initially excited, let them evolve to steady state, aiad repeat the calculation for an ensemble of initial conditions. We shall be content here with an investigation of the linear stability of the system. This problem has some intrinsic interest, but our 
main purpose is to lay the framework for an investigation of the mulzi-a system.

It is convenient to pose the linear stability problem in terms of the Fourier amplitudes (equations (13), (14) and (15)). We suppose that the system $w_{n}{ }^{\alpha}{ }_{1}, \Theta_{n}{ }^{\alpha}{ }_{1}$, and $B_{n}$ have their steady sta:e values, introduce disturbances $\delta \omega_{n}^{\alpha}, \delta \Theta_{n}$ and $\delta_{\beta_{n}}$ and ask wheither the latter grows or decays. Since $\alpha_{1}$ and $\alpha$ are not coupled, o $R_{n}$ must decay initially. The problem then is reduced to deterinining the growth rates for $\delta \mathrm{w}_{\mathrm{n}}^{\alpha}$ and $\delta \Theta_{\mathrm{n}}^{\alpha}$ in the presence of the mean gradient $B_{n}$. Since exchange of stabilities has been proved for this system ${ }^{10}$, we know that the system $w_{n}{ }^{\alpha}{ }_{1}, \Theta_{n}{ }^{\alpha}$, and ${ }{ }_{n}$ will. be stable if the smallest critical Rayleigh number $R_{c}$ for the perturbation system, $\delta w^{\alpha}, \delta \mathbb{A}^{\alpha}{ }_{n}$ is larger than the Rayleigh number for which $w^{\alpha}$ and $\oplus^{\alpha}$, were computed.

The marginally stable amplitude $\delta w_{n}^{\alpha}$, and $\delta \Theta_{n}^{\alpha}$ satisfy equations (13) and (15) at a Rayleigh number $R_{c}$, with the time derivatives put equal to zero. Since the smallest $R_{c}$ takes an eigen function even about $z=1 / 2$, we may abbreviate the perturbation system by eliminating the even sine modes from the velccity and temperature fluctuations. Defining

$$
\begin{aligned}
& \varphi_{n}=\delta w_{2 n-1} \\
& \bar{B}_{n}=B_{2 n},
\end{aligned}
$$

we may eliminate $\delta \Theta_{n}$ by using the steady state form of equation (13)' 
and write the marginally stability problem in the following matrix form:

$$
A(\bar{R}) \dot{\varphi}=\mu^{\varphi}
$$

where

$$
\begin{aligned}
& A(\bar{\beta})=\frac{\alpha^{2}}{\Gamma(2 n-1)^{2}+\alpha^{2} T^{3}}\left\{\delta_{n m}+\frac{1}{2}\left(\bar{\beta}_{\mid n-m !}-\bar{\beta}_{n+m-1}\right)\right\} \\
& \mu=\frac{\pi^{4}}{R_{c}}, \bar{\varepsilon}_{0}=0 .
\end{aligned}
$$

In writing the matrix $A$, we have used the alternative form for the convolution term in equation (14).

The largest eigen value, $j$ (smallest $R_{c}$ ), of equation (22) may be obtained by the matrix iteration technique (Hildebrand, 1952). Since the first sine mode of the velocity will be largest, we may convenicntly begin the iteration on a vactor containing only this mode. Defining

$$
11>=(1,0,0, \ldots, 0, \ldots)
$$

we may write

$$
\frac{\pi^{4}}{R_{c}(\alpha)}=u_{\max }=\lim _{n \rightarrow \infty} \frac{\left\langle 1\left|A^{n}\right| 1\right\rangle}{\left\langle 1\left|A^{n-1}\right| 1\right\rangle}
$$

The convergence of the iteration scheme is quite rapid because of the structure of the A matrix. At the highest Rayleigh number considered, $R=10^{6}$, the $11^{\text {th }}$ iteration gives $R_{c}$ to one part in $10^{6}$. 
The calculated $R_{C}(r)$ 's are shown in figure 17 for $R=5 \times 10^{5}$. The particular a which supports the mean field, $R$, labels the various curves. At low Ray leigh numbers, $\left(R<5.10^{3}\right)$ these curvos closely resemble those produced by perturbation calculations. Above $\mathrm{R} \sim 10^{5}$ they become increasingly distorted; steady statc amplitudes of small horizontal wave numbers are enormously unstable with respect to an introduction of a disturbance at large $\alpha$.

In figures (18) and (19) we give the zones of instability for the computed amplitudes for $R=10^{4}$ and $R=5 \times 10^{5}$. In these graphs, $\alpha_{1}$ is the wave number that supports the convective process, and $\alpha_{2}$ is the wave number of the perturbation amplitudes. The regions of instability are indicated by the shaded areas, whose outer boundaries are lines of marginal stability. The line $\alpha_{1}=\alpha_{2}$ is a trivial case of marginal stability. The value of a at which the two curves cross represents a solution which is infinitessimally stable against all other $a^{\prime} s$. This value of $a$ begins at $1 / \sqrt{2}$ at the critical Rayleigh number and increases slowly with increasing $R$. The rate of increase is seen to be slower than that $\alpha$ which maximizes the total heat transport. Referring to figure (15) we see that the use of the most stable $\alpha$ instead of $\alpha_{\max }$ will not apreciably change the total heat transport.

The zones which linear stability theory predict must have two or more a's supporting convection are indicated by the cross riatched regions in figures (18) and (19). These regions are obtained by perturbing the $\alpha_{1}$ fields at $\alpha_{2}$, assuming that the $\alpha_{2}$ field 
subsequently dominates the convection, and then demanding that the $\sigma_{2}$ fields be unstable with respect to a perturbation at $r_{1}$. The cross-hatched region is then bounded by the descending marginally stable curve and its reflection about the $45^{\circ}$ line. At small Rayleigh number, $R<4 \times 10^{3}$, this area vanishes but it gradually increases with Rayleigh number.

Concluding Remarks

The temperature and velocity fields computed here with the fluctuating self-interactions absent show qualitatively a reasonable behavior: The boundary-layering of the temperature field, which is found experimentally, is faithfully reproduced by the system, and the heat transport has the experimentally detcrmined dependence on Rayleigh number. In this respect, our results for the velocity and temperature amplitudes, as well as the stability analysis of the fields, confirm the original ideas of Malkus. However, our result for the heat transport for free boundary conditions does not agree quantitatively with Malkus.

The only disquieting features of the results are the negative temperature gradients which can occur near the boundary for small $a$, and the rather large amount of heat transported by the system.: Aside from eddy processes, there are two other modifications in the system which must be explored before its quantitative accuracy can se properly accessed.

First, the use of the more realistic rigid boundary conditions will enable one to examine quantitatively the role of the ecing rocosses in producing the experimental temperature profile and the total heat flux. The presence of shear forces at the boundary will decrease the 
computec heat flux, and in checking the development of large scale horizontal motions there, it will reduce the negative temperature gracient. Preliminary indications aic that the use of rigidbourdary conditions decrease the total heat transport by a factor of 2.3. Secondly, the introduction of several horizontal wave numbers will make the system more realistic, particularly at large Rayleigh numbers. It will also permit a study of finite amplitude stability of the system. The above modifications are currently under investigation and will be reported on in the near future. 


\section{ACKNOWLEDGEMENTS}

The author is very grateful to Dr. R. H. Kraichnan for suggesting this investigation, for many illuminating discussions through the completion of the work, and for making helpful suggestions regarding the presentation of material. He is also grateful to Dr. E. A. Spiegel for many valuable discussions, and for critically reading a final version of the manuscript.

It is a pleasure to acknowledge the able assistance of Mr. R. Rustin, who programmed and carried out the numerical conputations on the IBM 7090 computer. 
FOOTNOT

1. For a discussion of the PrandtI number dependence of the heat transport for the complete system see Kraichnan, R. H., 1962: Turbulent Thermal Convection at Arbitrary Prandtl Number. Physics of Fluids 5 1374-1389.

2. See e.g. Chandrasekhar, S., 1961: Hydrodynamic and Hydromagnetic stability. Oxford at the clarendon press, p. J.6.

3. Discarding third order cumulants is quite different from discarding third order moments. The latter procedure has as a consequence that no steady state nontrivial amplitudes exist. For an investigation of the dynamics of decay for zero thirdorder moments see Deissler, R. G., 1962: Turbulence in the Presence of a Vertical Body Force and Temperature Gradient, J. Geophys. Research, 67, 3049-3062.

4. For a complete discussion of the cumulant discard approximations see Kraichnan, R. H., 1962. The Closure Problem of Turbulence Theory, Proceedings of Symposia in Applied Mathematics, Vol. 13. Hydrodynamic Instability, American Mathematical Society, 199-225.

5. See Malkus and Veronis, loc. cit., p. 228 for a complete discussion of the conservation equations.

6. See Chandrasekhar, 10c. cit., p. 35.

7. Spiegel, loc. cit., p. 196. 
8. Malkus, loc. cit., p. 200.

9. This fact has the consequence that the heat transport, $N_{\max }$ i: asymptotically proportional to $\mathrm{R}^{3 / 10}$. R. H. Kraichnan, p:ivate communication.

10. Spiegel, loc. cit. p. 196. 
REFERENこES

1. Jakob, M., 1959: Heat Transfei V.I. I. John Wiley and Sons, InC. New York, p. 535

2. Hildebrand, F. B., 1952: Methods in Applied Mathematics, Prentice-Hall, Inc., Englewood Cliffs, New Jersey, p. $66^{\circ}$. .

3. Kuo, H. L., 1961: Solution of the Nonlinear Equations of Cellular Convection and Heat Transport. ' $\mathrm{J}$. Fluid Mech. 10 .' 611-634.

4. Malkus, W. V. R., 1954: The Heat Transport and Spectrum of Thermal Turbulence. Proc. Roy. Soc. London, A, 225, 196-212.

5. Malkus, $\dot{W}$. V. R., 1960: Turoulence, Notes of Summer Study Program in Geophysical Fluid Dynamics at the Woods Hole Oceanographic Institution, Unpublished, p. 38 et seq.

6. Malkus, W. V. R. and G. Veronis, 1958: Finite Amplitude Cellulax Convection. I. Fluid Mech. 4, 225-260.

7. Ogura, Y., 1962: Energy Transfer in an Isotropic Turbulent Fjow. J. Geophys. Research, 67, 3143-3150.

8. Saltzman, B., 1961: Finite Amplitude Free Convection as an Initial Value Problem. J. Atmos. Sciences 19, 329-341.

9. Spiegel, E. A., 1962: On the Malkas Theory of Turbulence Mechanique de la Turbulence C. N. R. S. Paris 181-201. 


\section{Captions}

Eigure 1. Time development of the total heat flux, $N(\tau)$ for

$R=4 \times 10^{3}, 10^{4}, 10^{5}$ and $\alpha-1.5$. The system is in the conduct.jon state at $T=0$, with all fluctuating amplitudes except $w_{1}$ equal to zero.

Figure 2. $4.22 \times 10^{-2} \mathrm{~W}$ and $4.07 \Theta$ for $R=4 \times 10^{3}$ and $\alpha=0.8$. Figure $3(a)$ Mean Temperature, $\bar{T}(z)$, for $R=4 \times 10^{3}$ and $\alpha=0.8$. Eigure $3(b)$ Mean gradient, $B(z)$, for $R=4 \times 10^{3}$ and $\alpha=0.8$. $\beta(z)$ is normalized by the total heat transport, $N=3.92$. Figure 4. $2.05 \times 10^{-2} \mathrm{w}$ and $5.16 \Theta$ for $R=10^{4}$ and $\alpha=1.0$. Figure $5(a)$ Mean Temperature, $\bar{T}(z)$, for $R=10^{4}$ and $\alpha=1.0$. Figure 5(b) Mean gradient, $\beta(z)$, for $R=10^{4}$ and $\alpha=1.0$. $\beta(z)$ is normalized by the total heat transport, $N=5.82$. Figure 6. $4.33 \times 10^{-3} \mathrm{~W}$ and $9.42 \Theta$ for $R=10^{5}$ and $\alpha-1.5$. Figure 7(a) Mean Temperature, $\bar{T}(z)$, for $R=10^{5}$ and $\alpha=1.5$. Figure $7(b)$ Mean gradient, $B(z)$, for $R=10^{5}$ and $\alpha=1.5$. $\beta(z)$ is normalized by the total heat transport, $N=13.82$. Figure 8. $8.98 \times 10^{-4} \mathrm{~W}$ and $19.4 \oplus$ fo:: $R=10^{6}$ and $\alpha=1.5$. Figure $9(a)$ Mean Temperature, $\bar{T}(z)$ for $R=10^{6}$ and $\alpha=1.5$. Figure $9(b)$ Mean gradient, $B(z)$, : or $R=4 \times 10^{6}$ and $\alpha=1.5$. $\beta(z)$ is nomalized by the total heat transport, $N=31.48$. Figure $10 . \quad 3.22 \times 10^{-3} \mathrm{w}$ and $8.57 \Theta$ for $\mathrm{R}=10^{6}$ and $\alpha=6.0$. Eigure $11(a)$ Mean temperature, $\bar{T}(z)$, for $R=10^{6}$ and $\alpha=6.0$. Figure $11(b)$ Mean gradient, $\beta(z)$, for $R=10^{6}$ and $\alpha=6.0$. $\beta(z)$ is normalized by the total heat transport, $N=22.3$. 
Figure 12. $1.06 \times 10^{-2} \mathrm{w}$ and 12.90 for $\mathrm{R}=10^{6}$ and $\alpha=9.0$. Figure 13(a) Mean temperature, $\bar{T}(z)$, for $R=10^{6}$ and $\alpha=9.0$. Figure 13(b) Mean gradient, $\beta(z)$, for $R=10^{6}$ and $\alpha=9.0$. $\beta(z)$ is normalized by the total heat transport, $N=5.40$. Figure 14 . Cosine spectrum of the mean temperature gradient for $R=10^{4}, 10^{5}, 10^{6}$ and $\alpha=1.5$.

Figure 15. The total heat transport $\mathrm{N}$ as a function of a $r$; $R=4 \times 10^{3}, 10^{4}, 10^{5}, 5 \times 10^{5}$ and $10^{6}$. Figure 16. Maximum total heat transport, $\mathrm{N}_{\max }$ as a function of $\mathrm{R}^{1 / 3}$.

Figure 17. Critical Rayleigh number $R_{C}$ for $R=5 \times 10^{5}$ as a function $\alpha$. The value of $\alpha$ which supports the mean temperature fields labels the various curves. Figure 18. Stability diagram for $R=10^{4}, \alpha_{1}$ is the wave number that supports convection, and $\alpha_{2}$ is the wave number at which a small perturbation is introduced. The shaded region indicates instability.

Figure 19. Stability diagram for $R=5 \times 10^{5} \cdot a_{1}$ is the wave number that supports convection, cnd $\alpha_{z}$ is the wave number at wh ich a small perturbation is introduced. The shaded regions irdicate instability: 


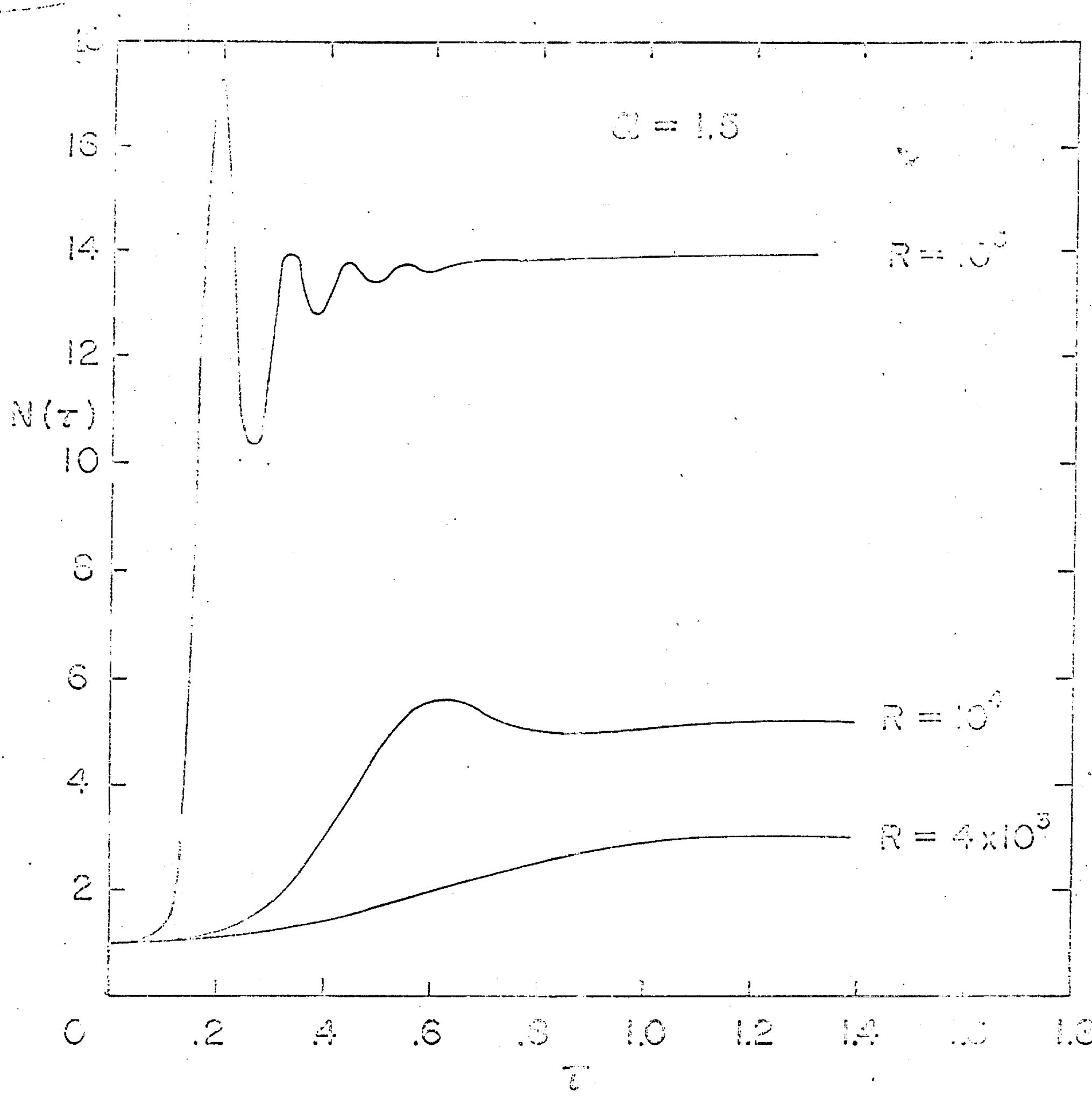




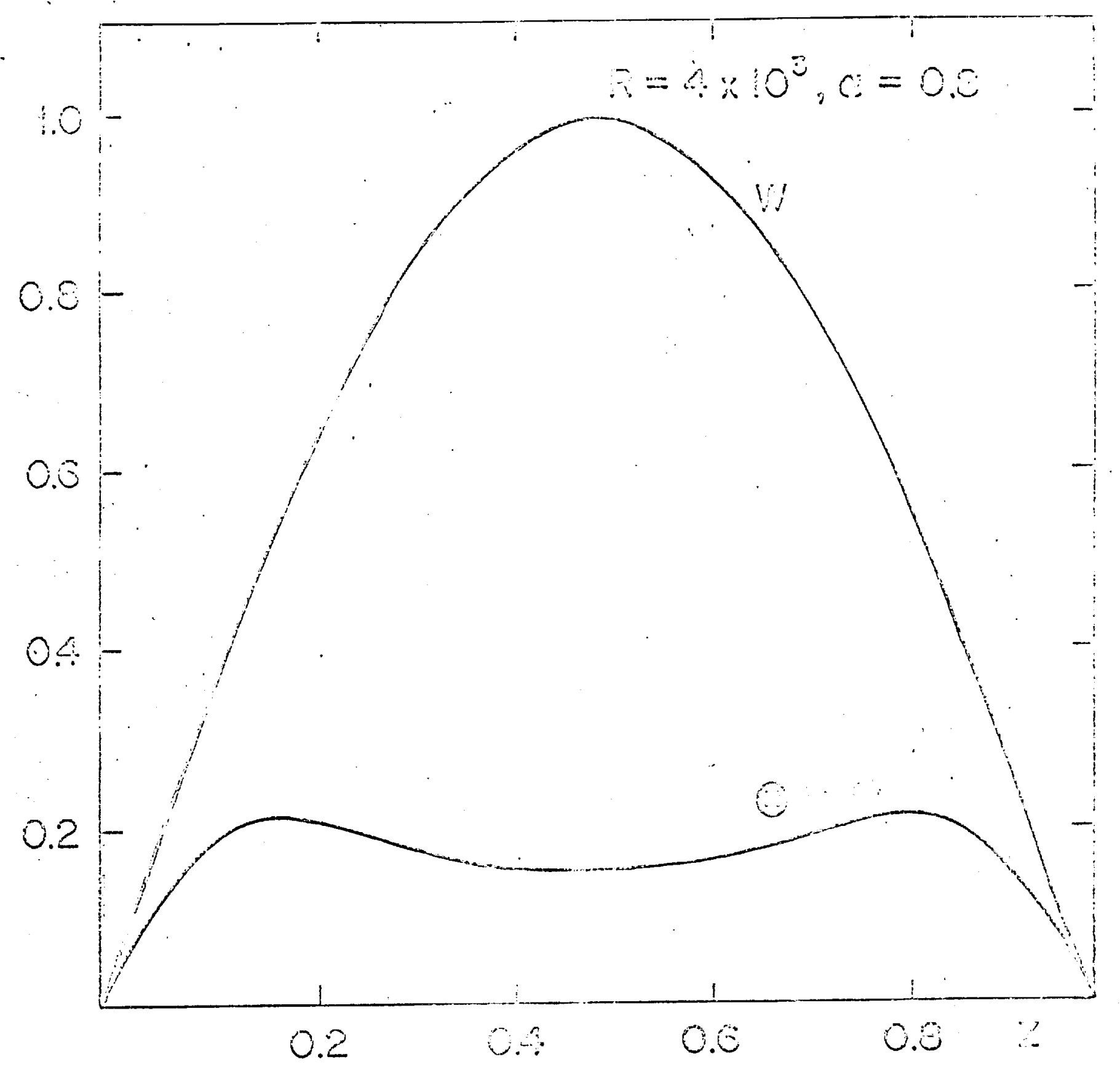




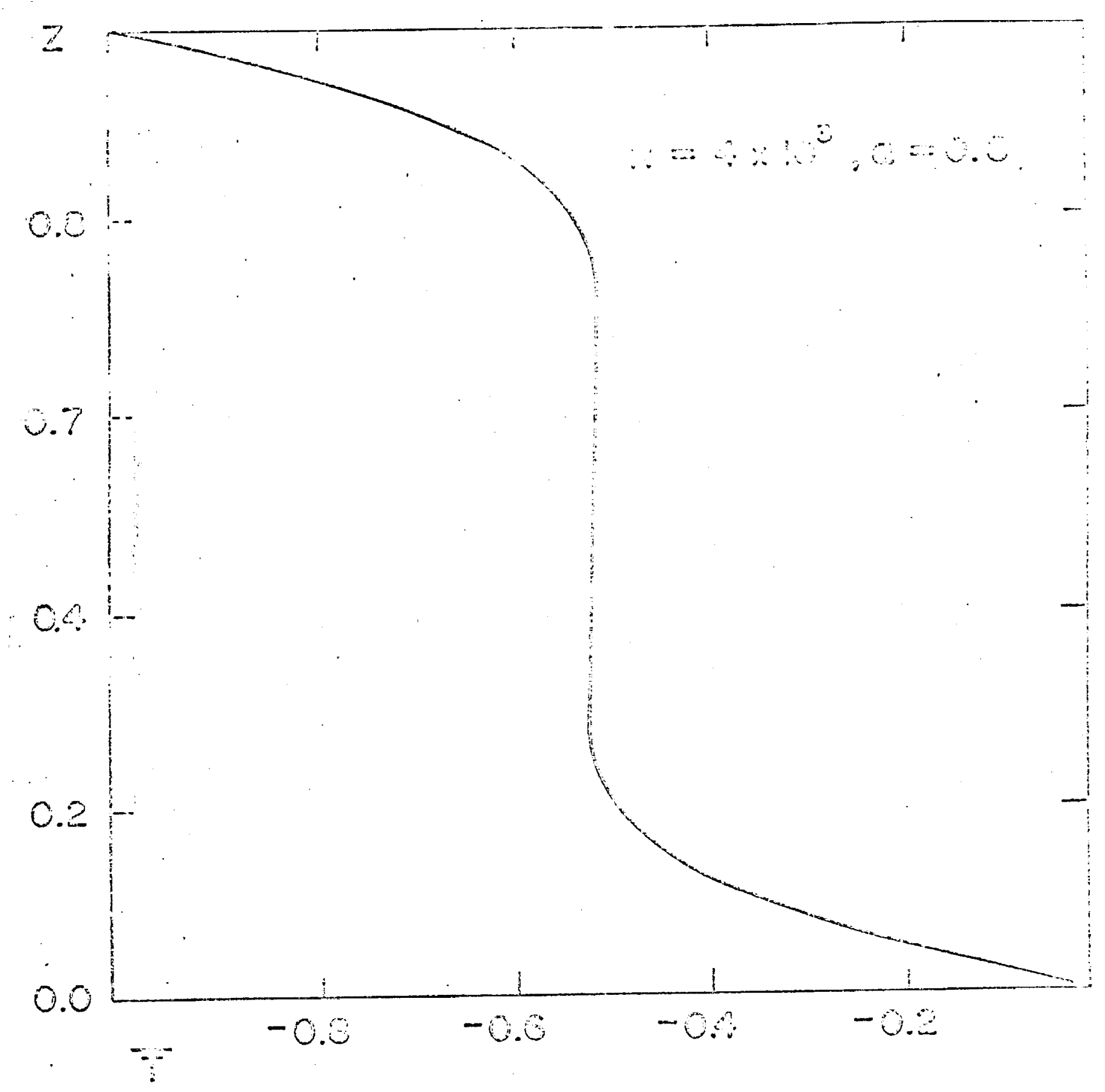




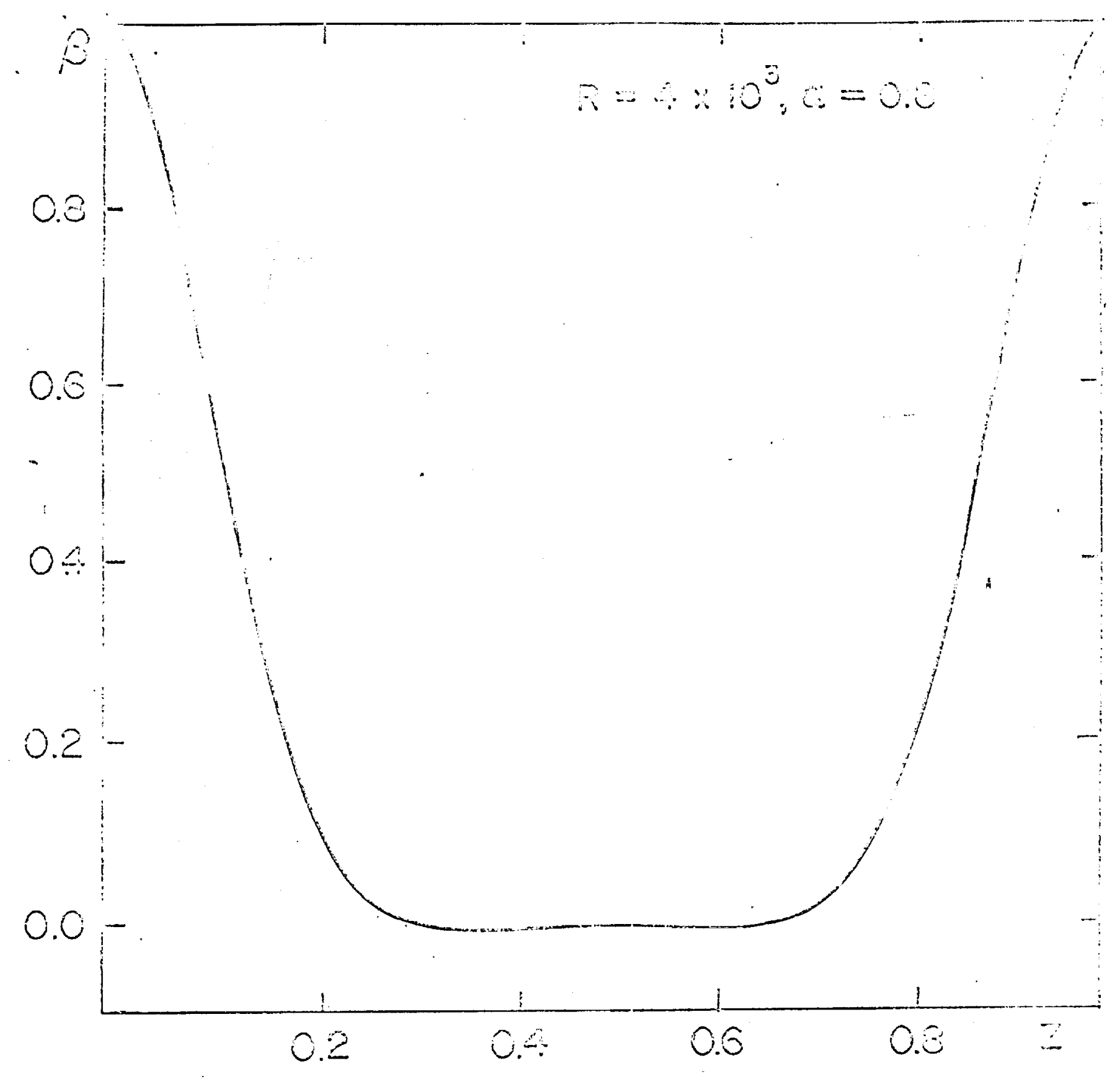




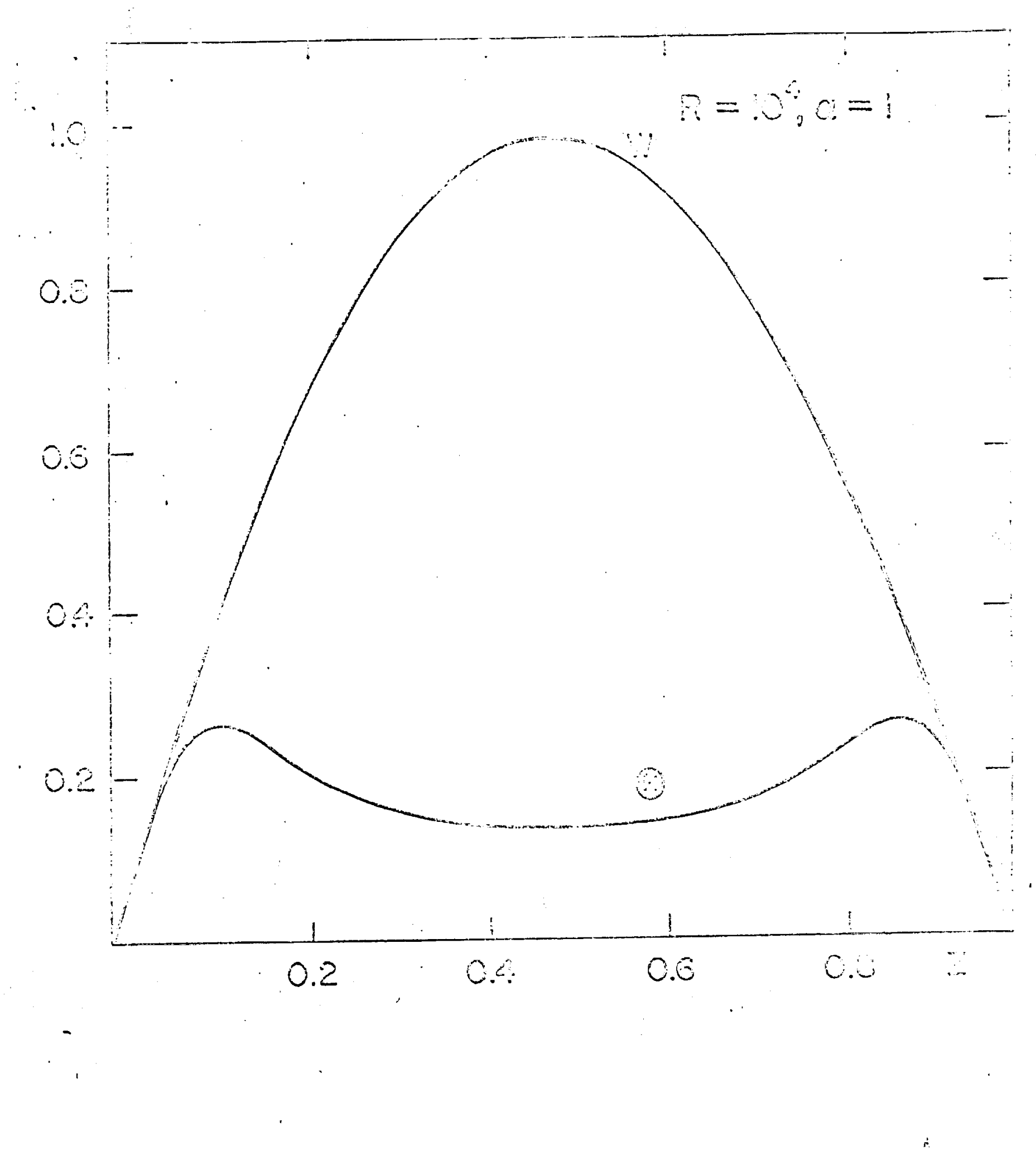




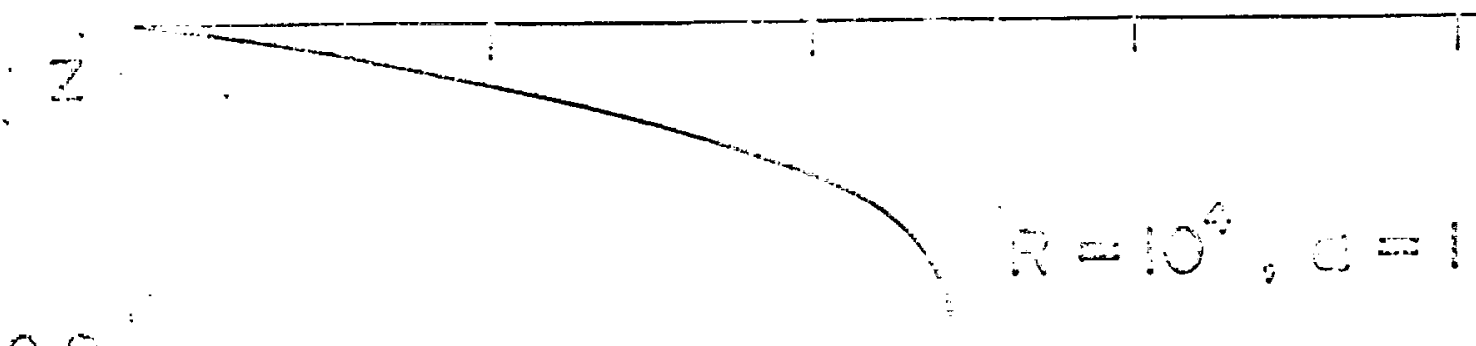

$C^{-}$

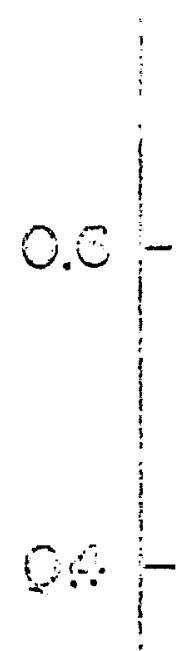

$\div$

$\vdots$

0.2
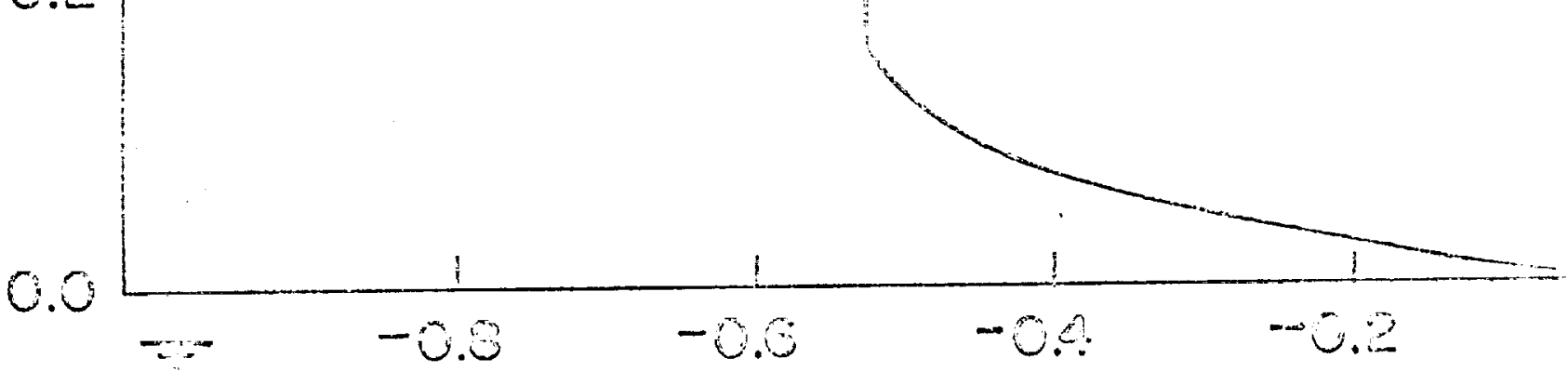

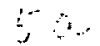


F

$$
\therefore=O Q \because, C=!
$$

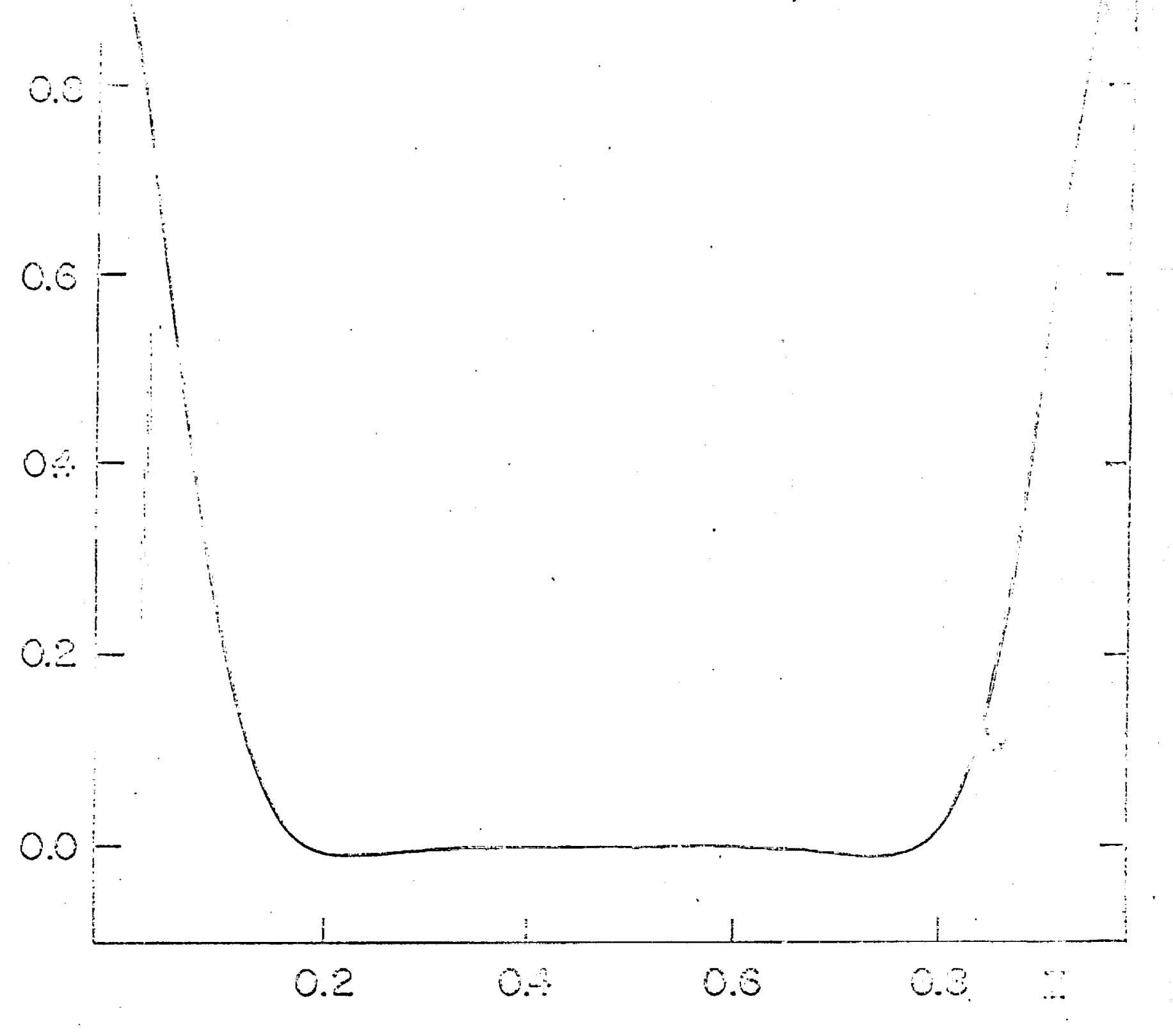




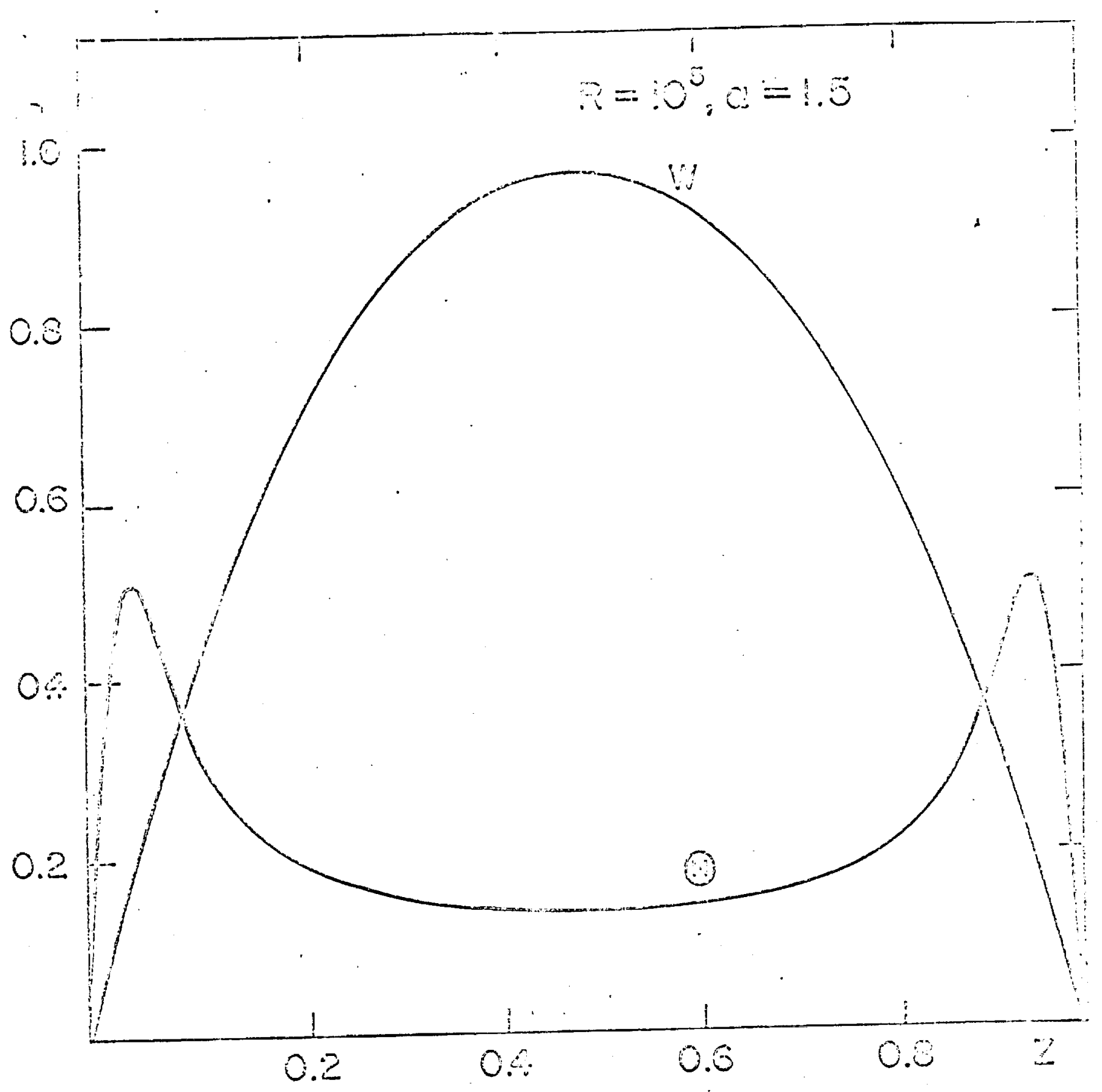

(1)

n. 


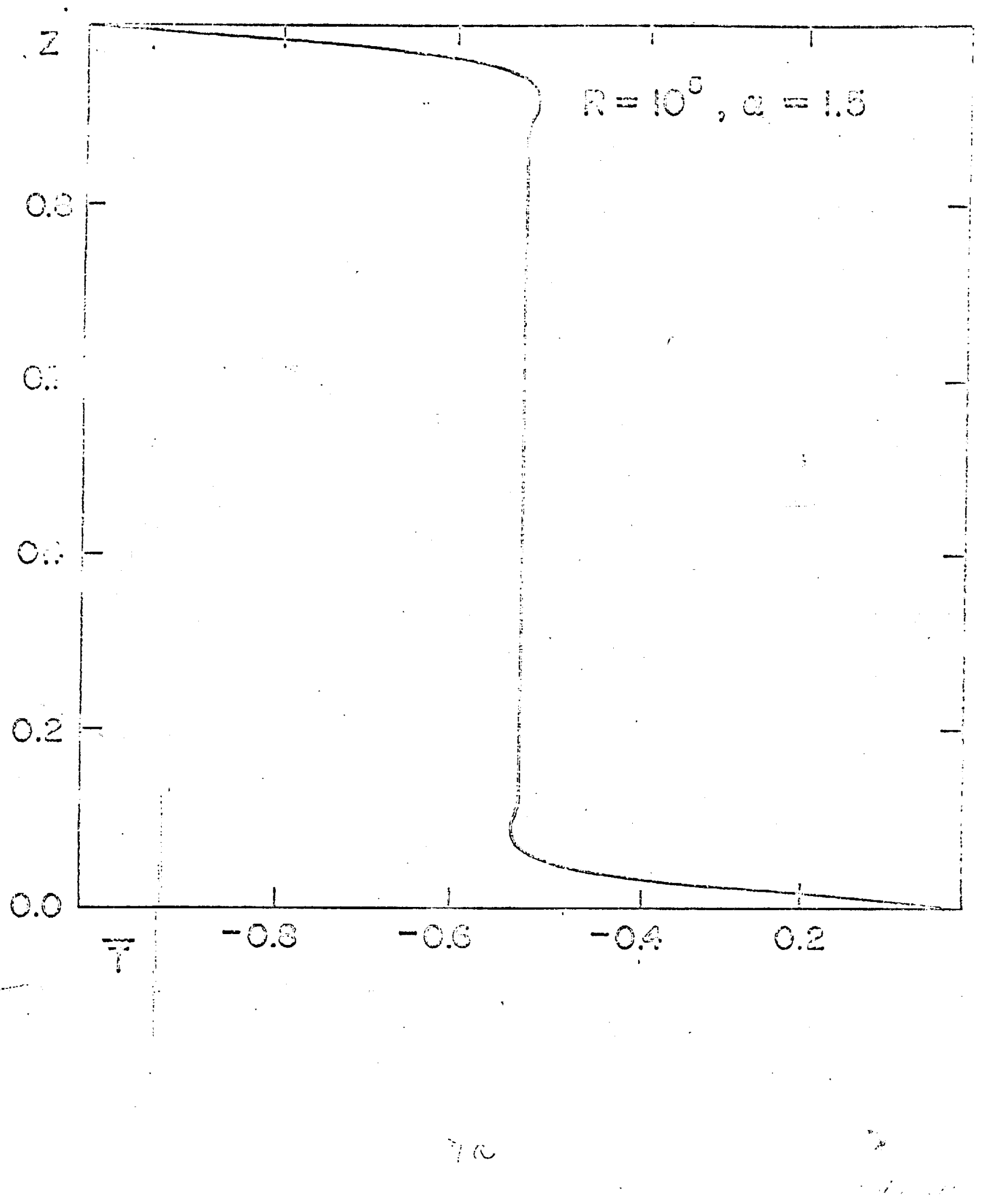



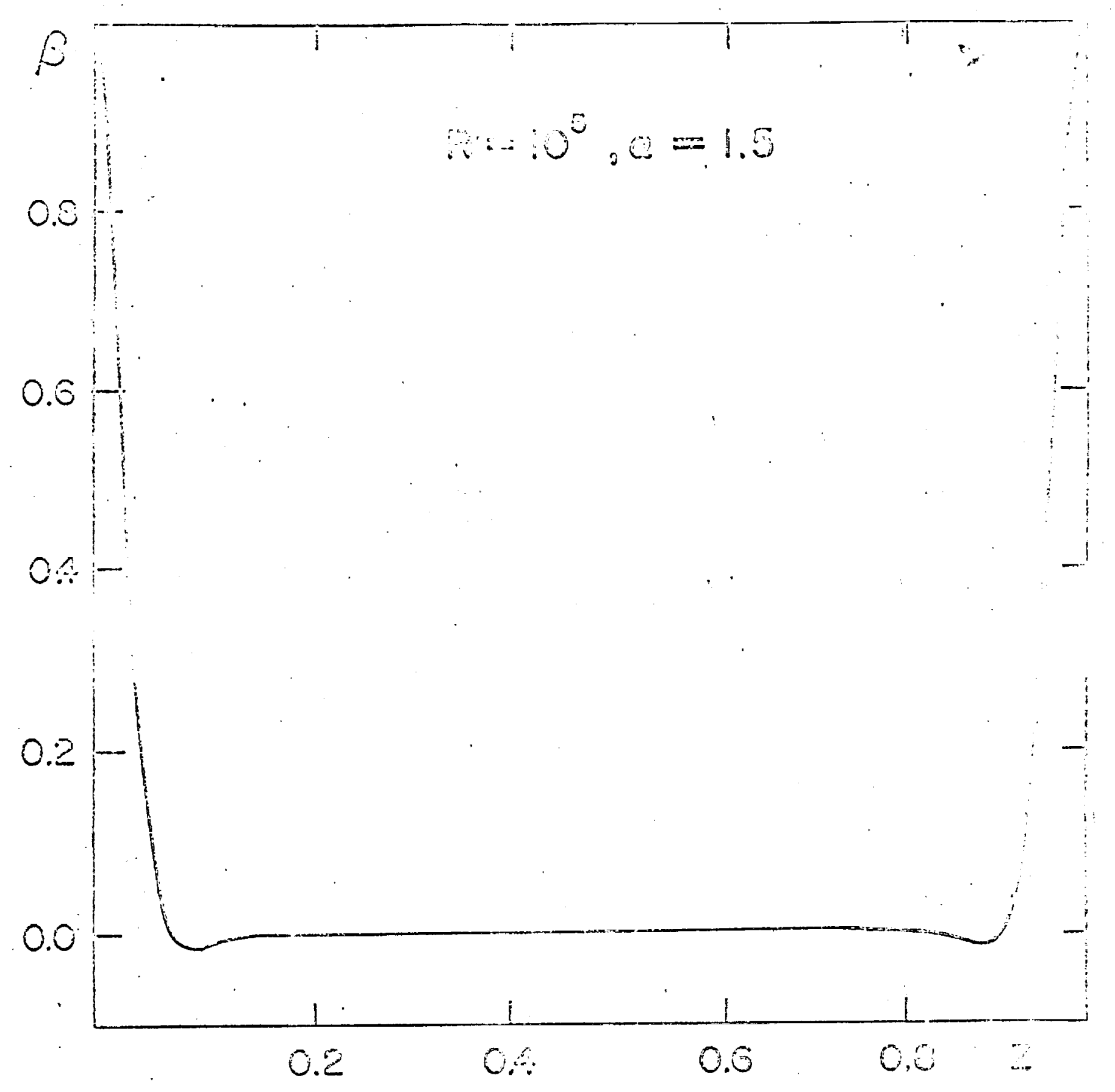

i 1. 

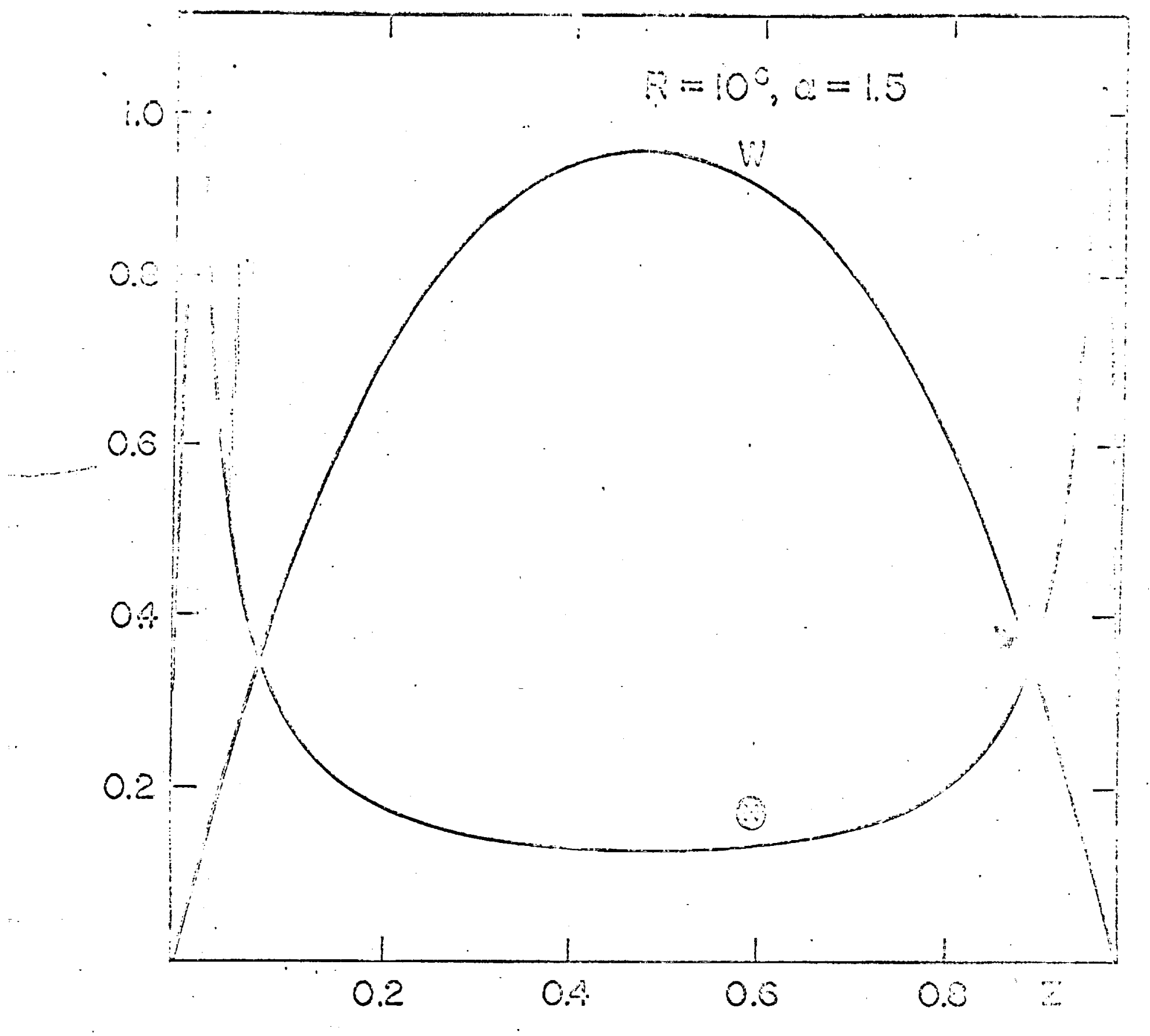


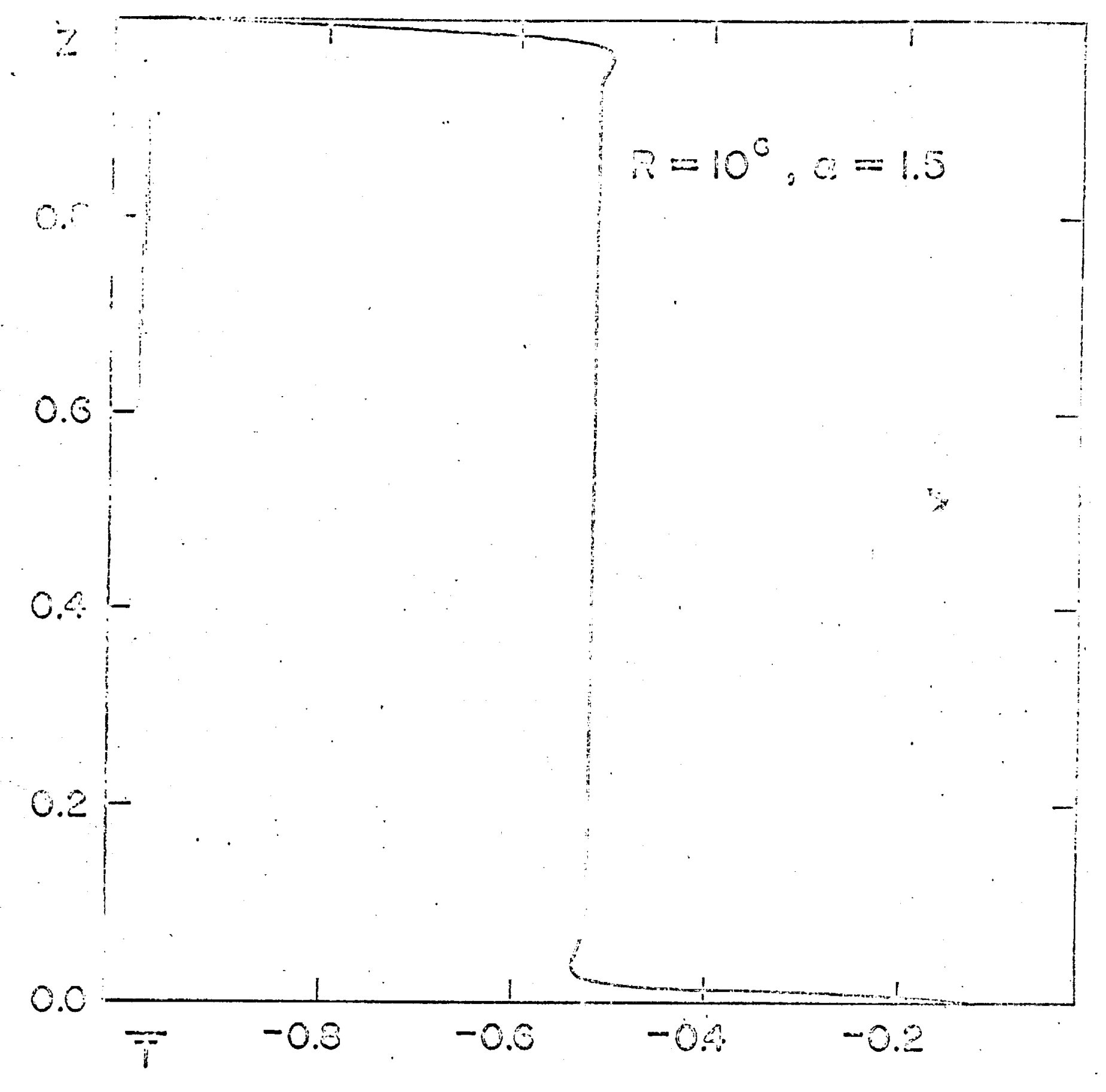

qa 


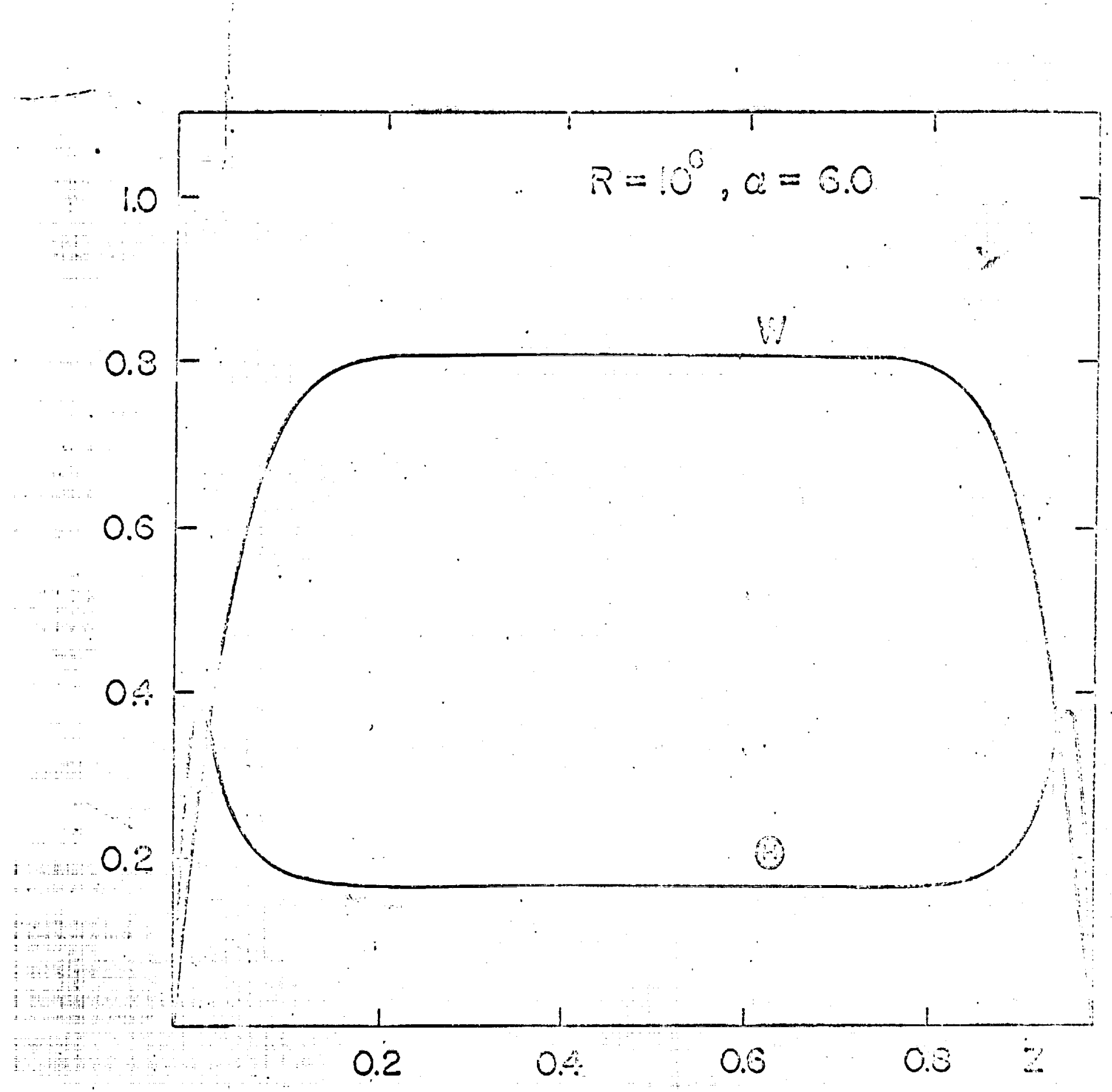




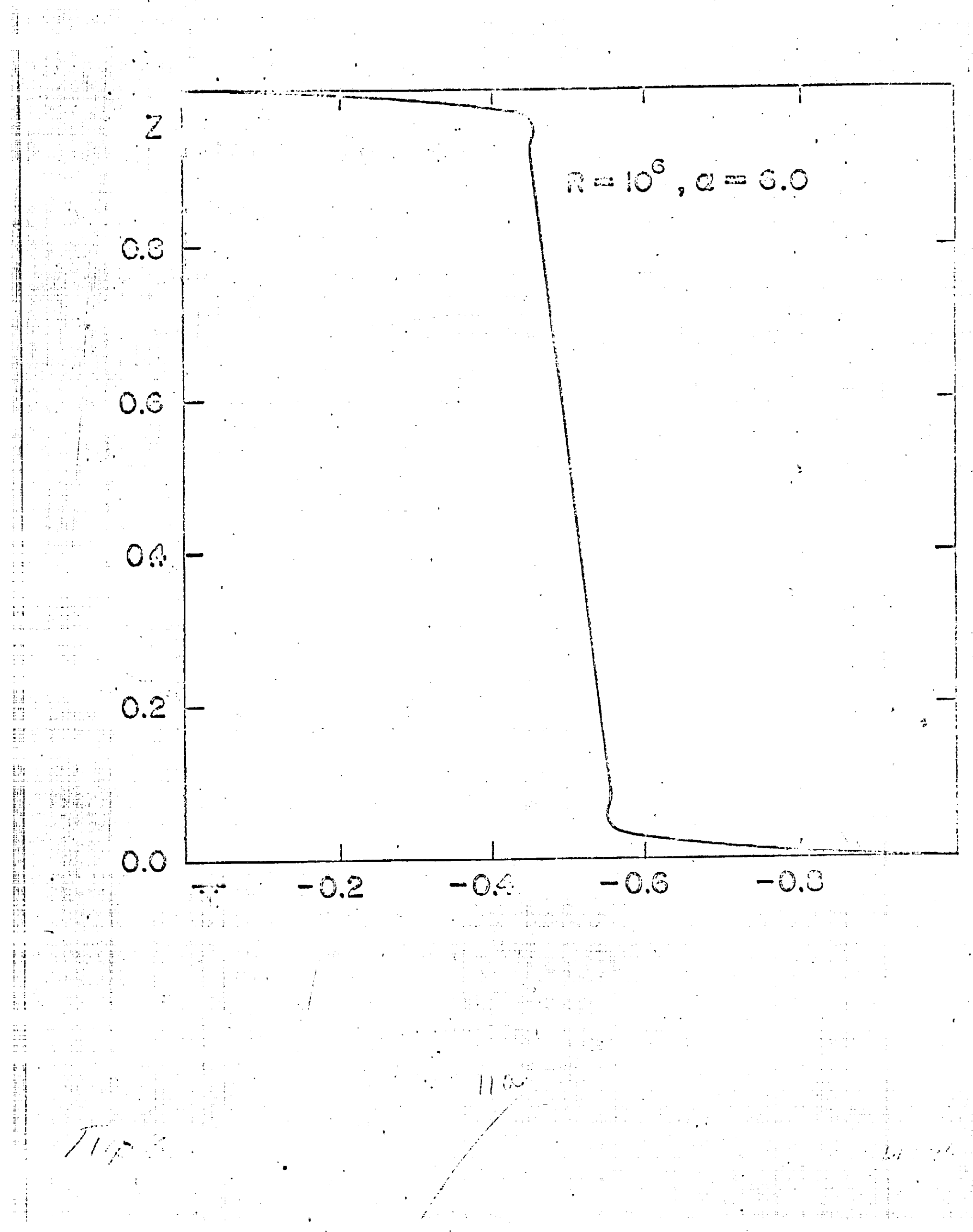




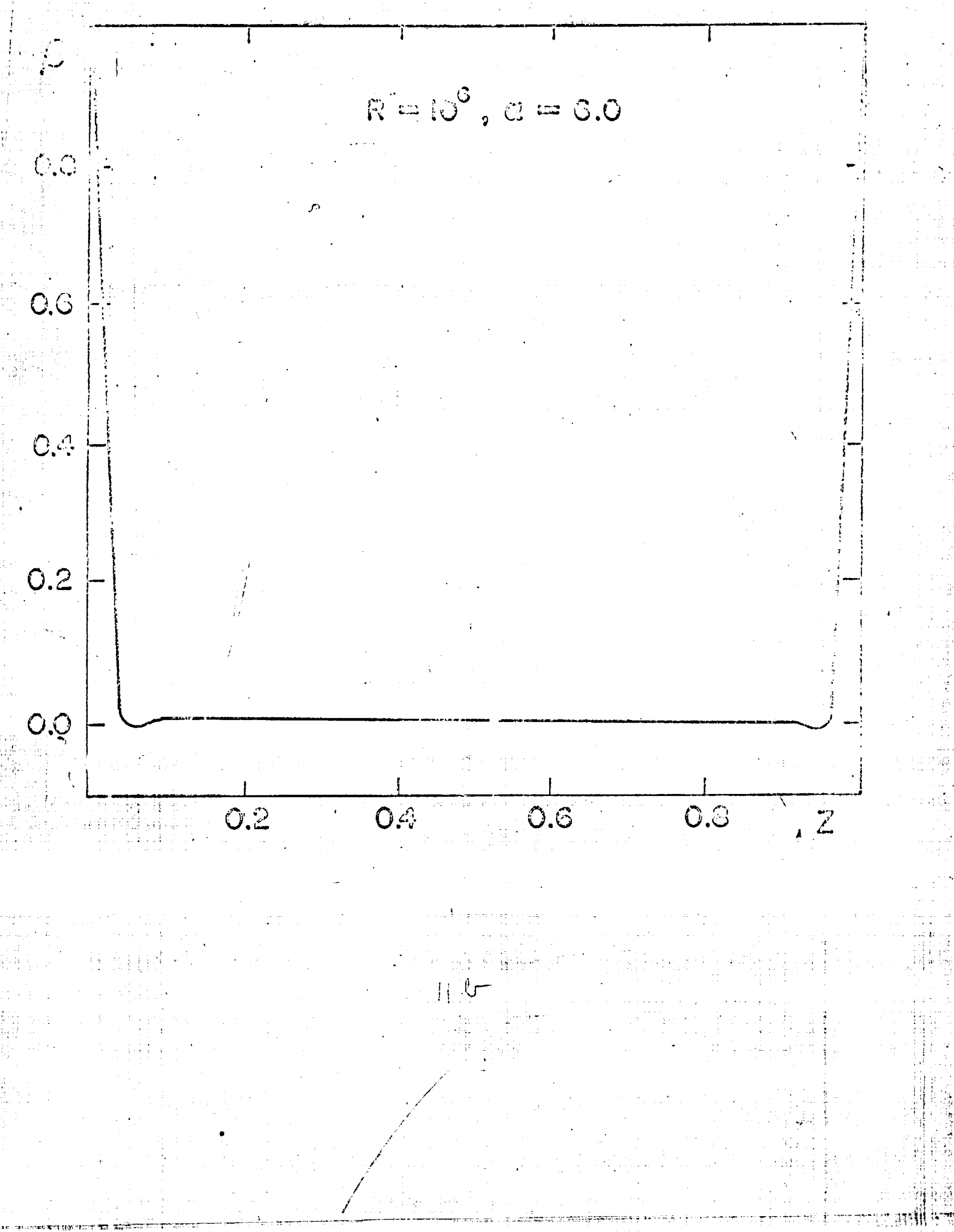




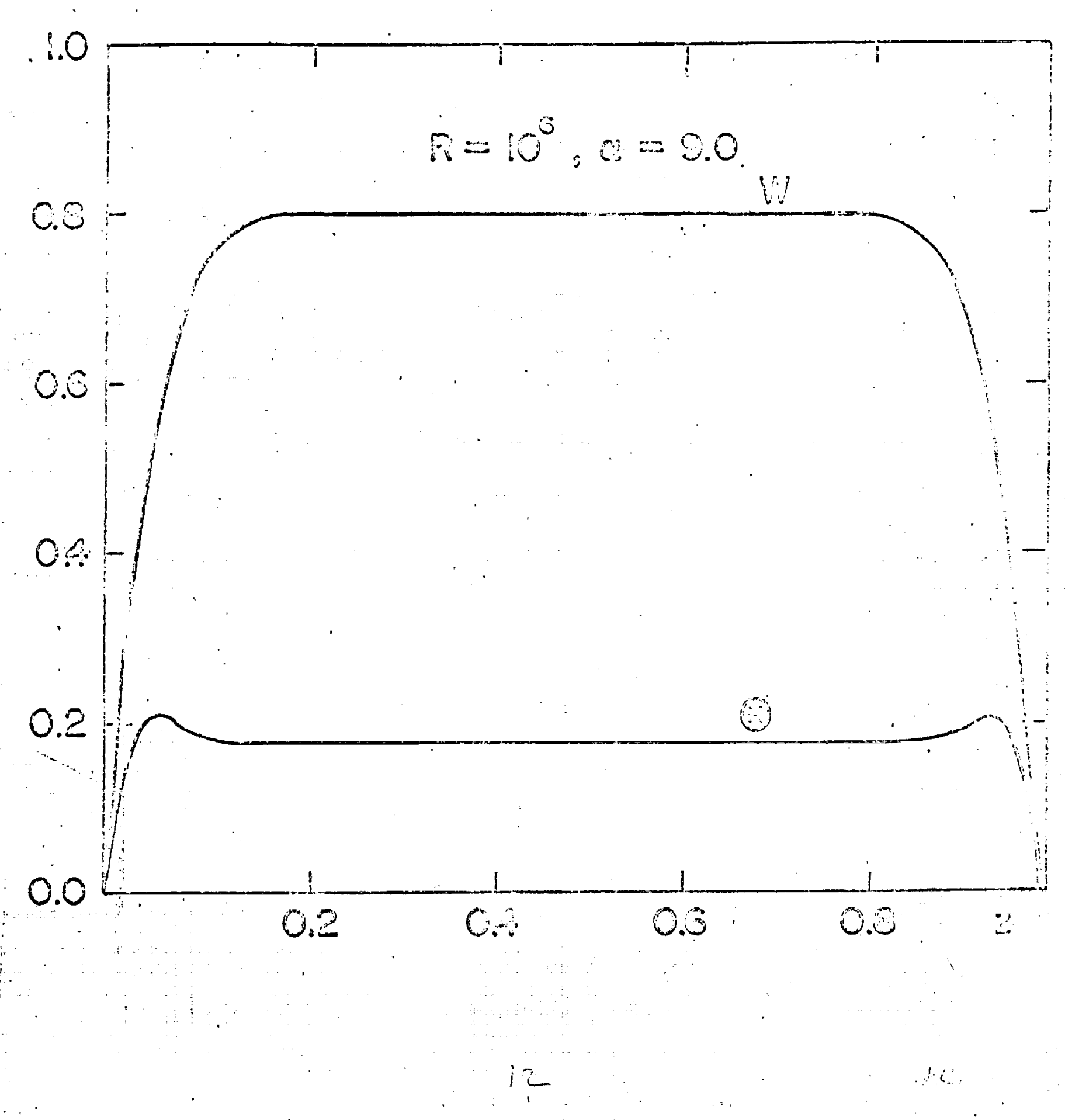




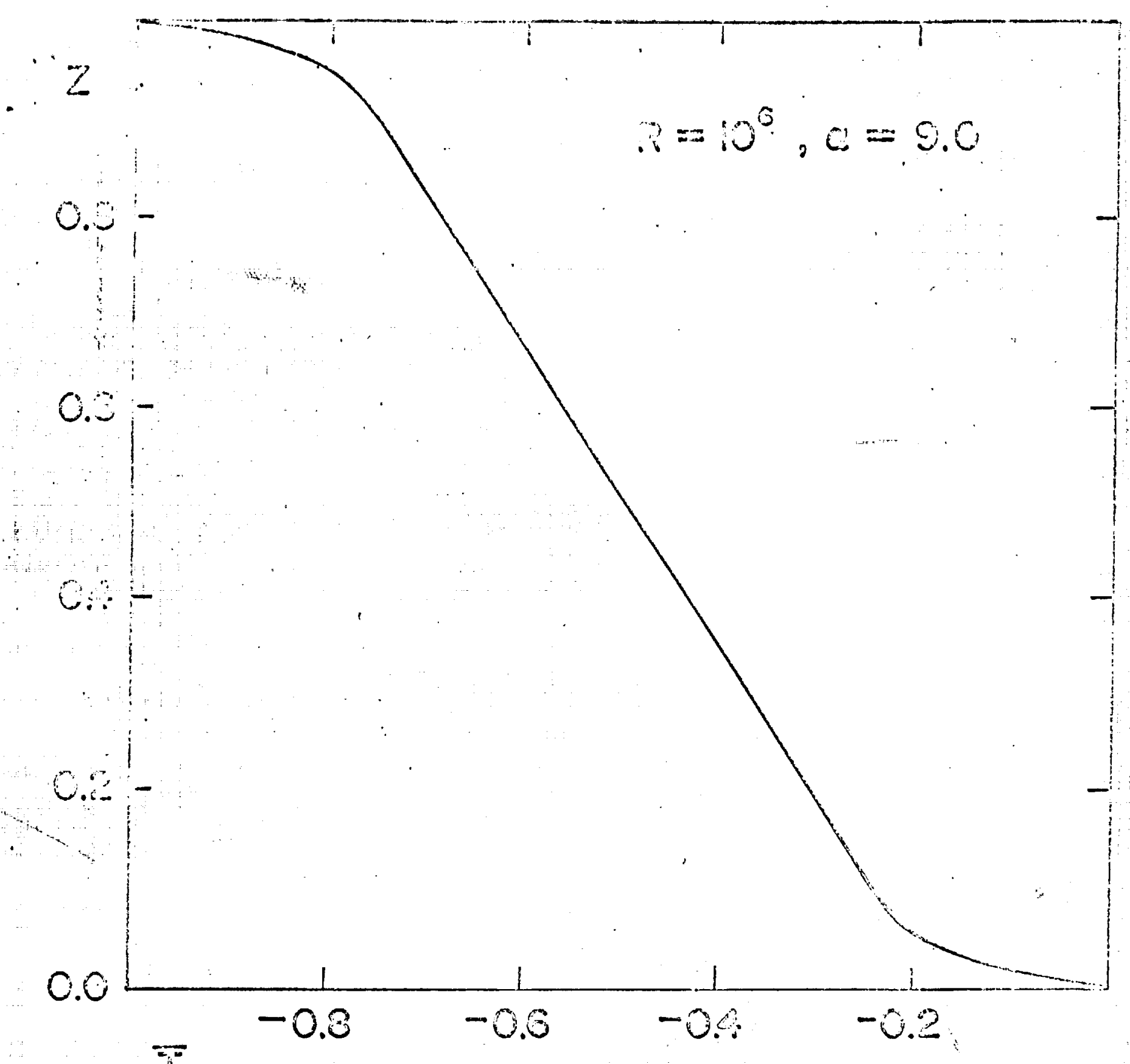

.132 


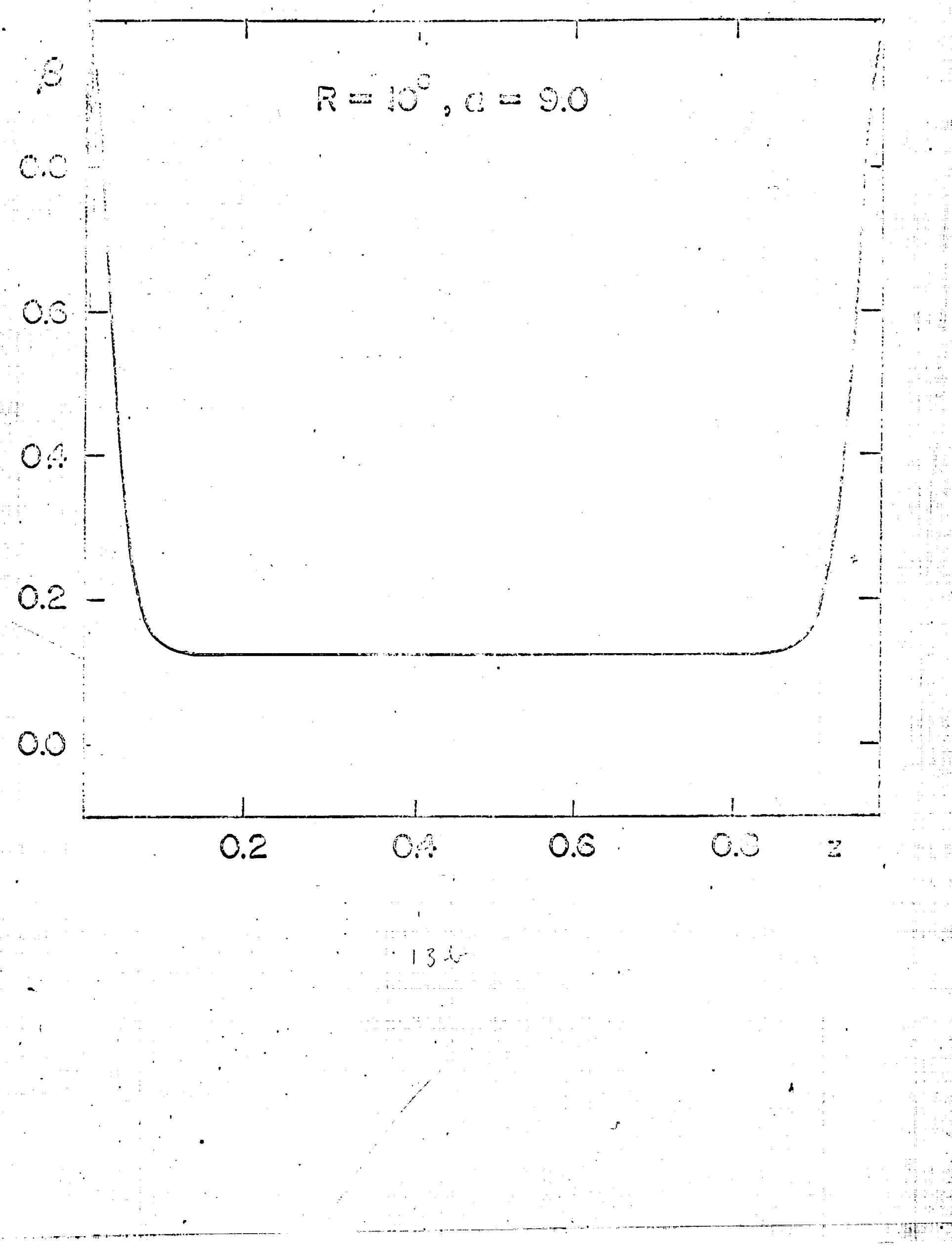




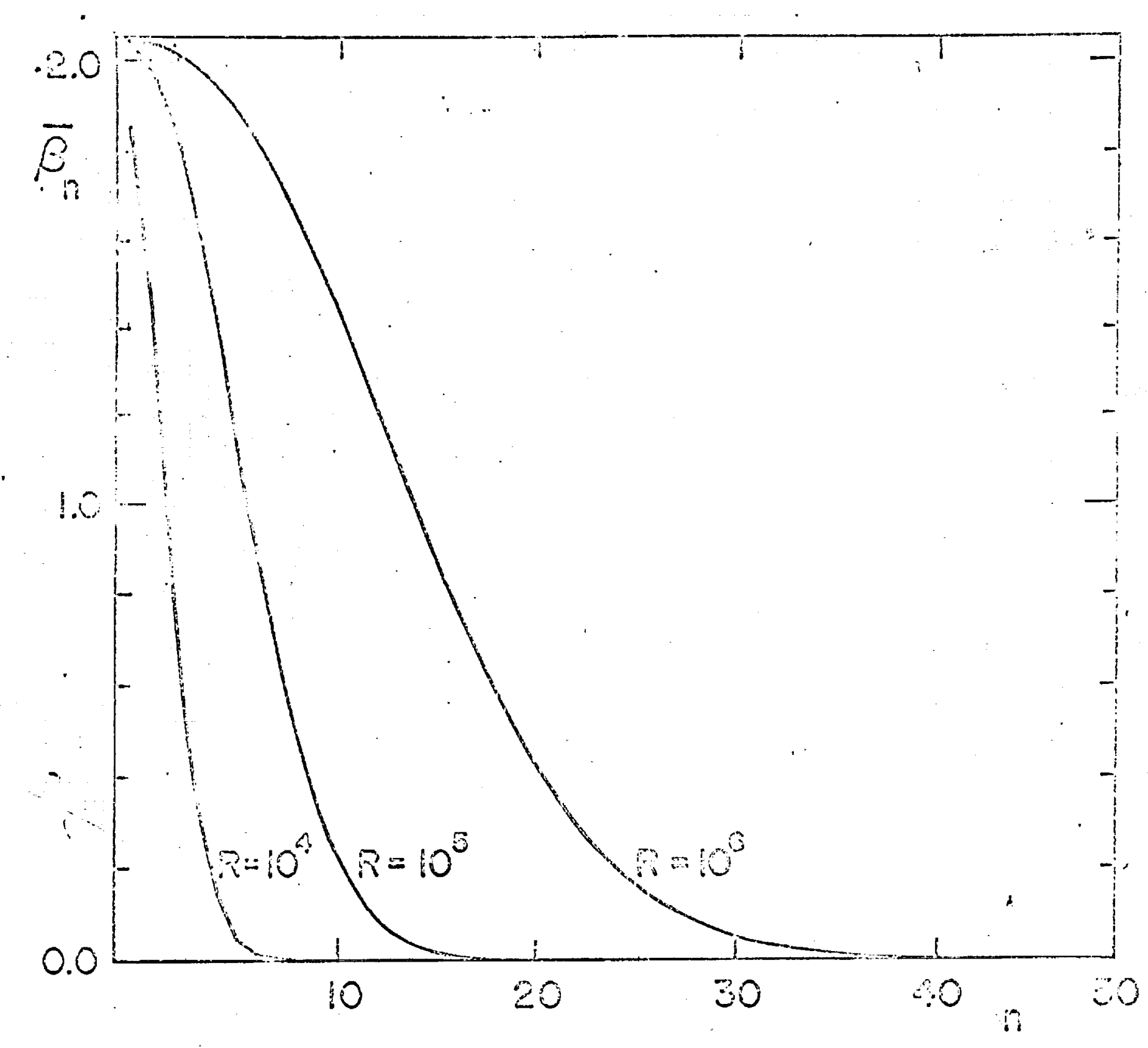




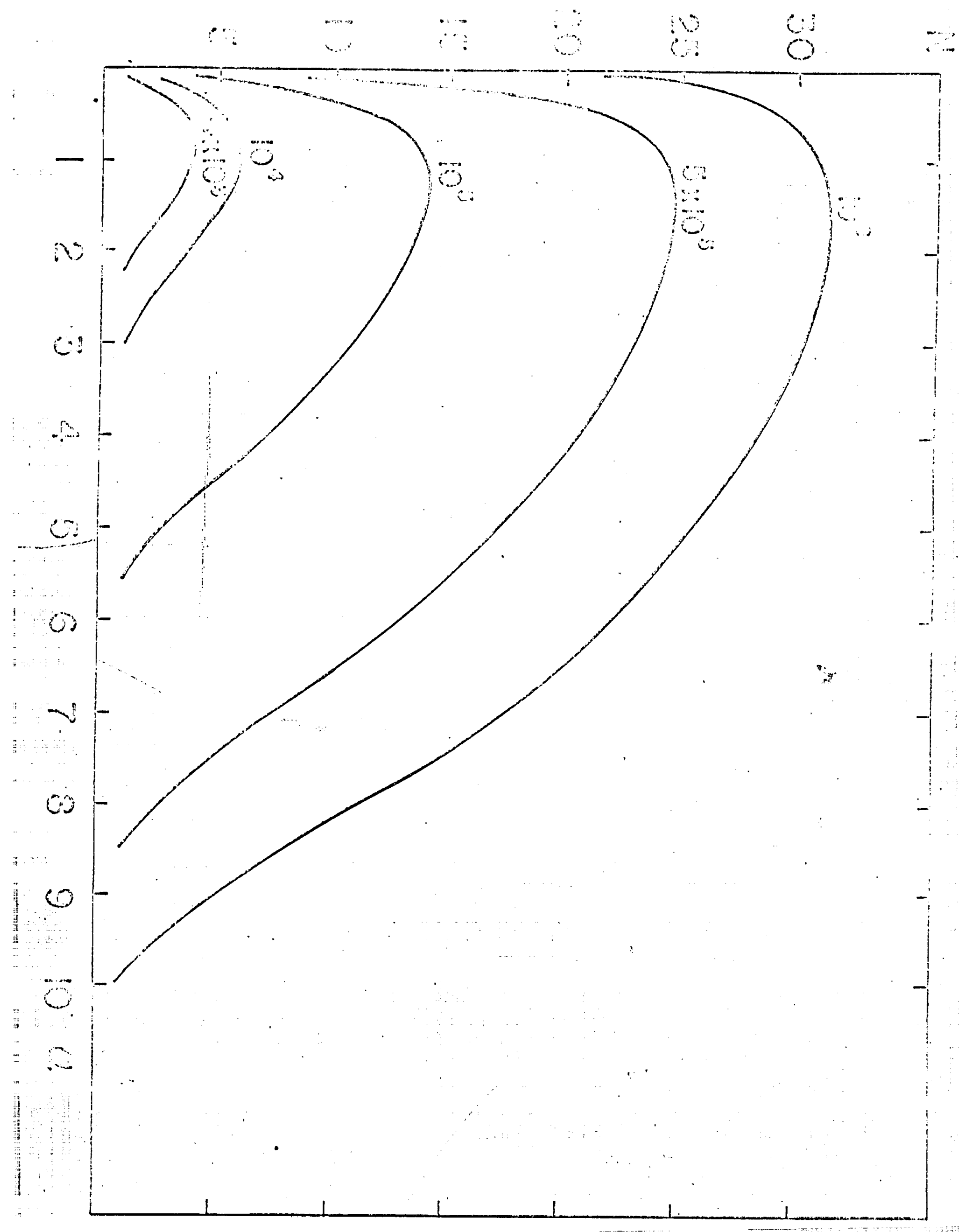




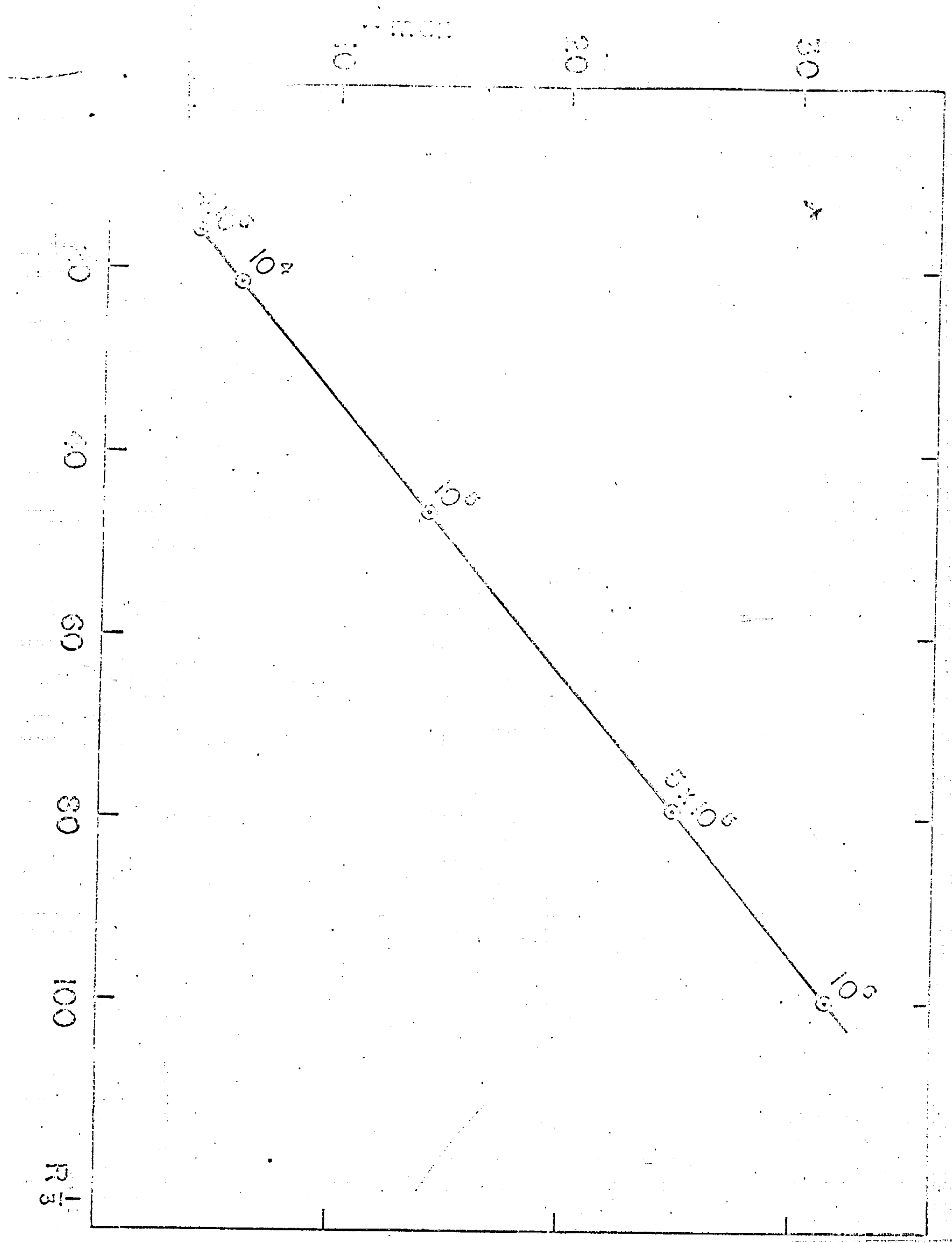




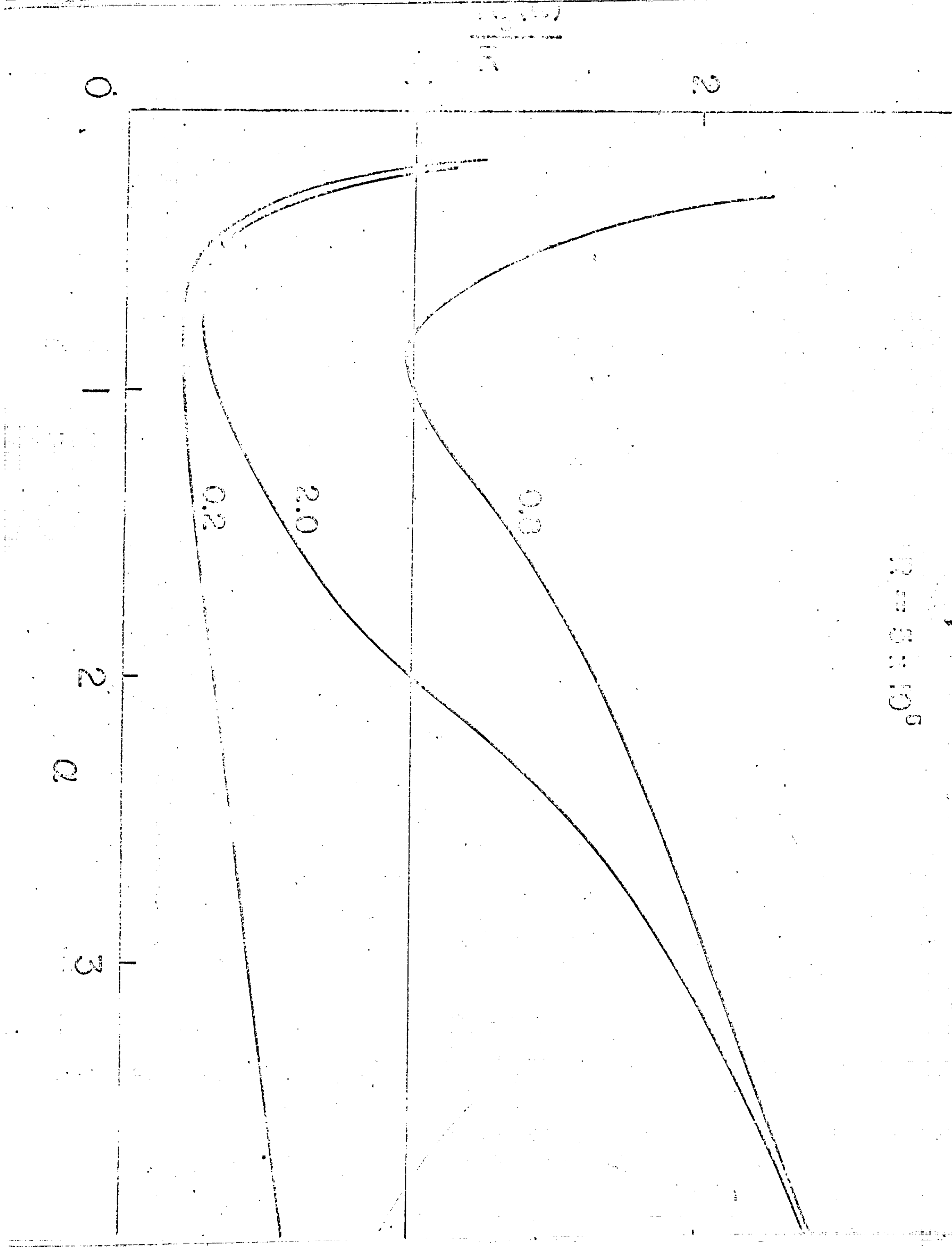




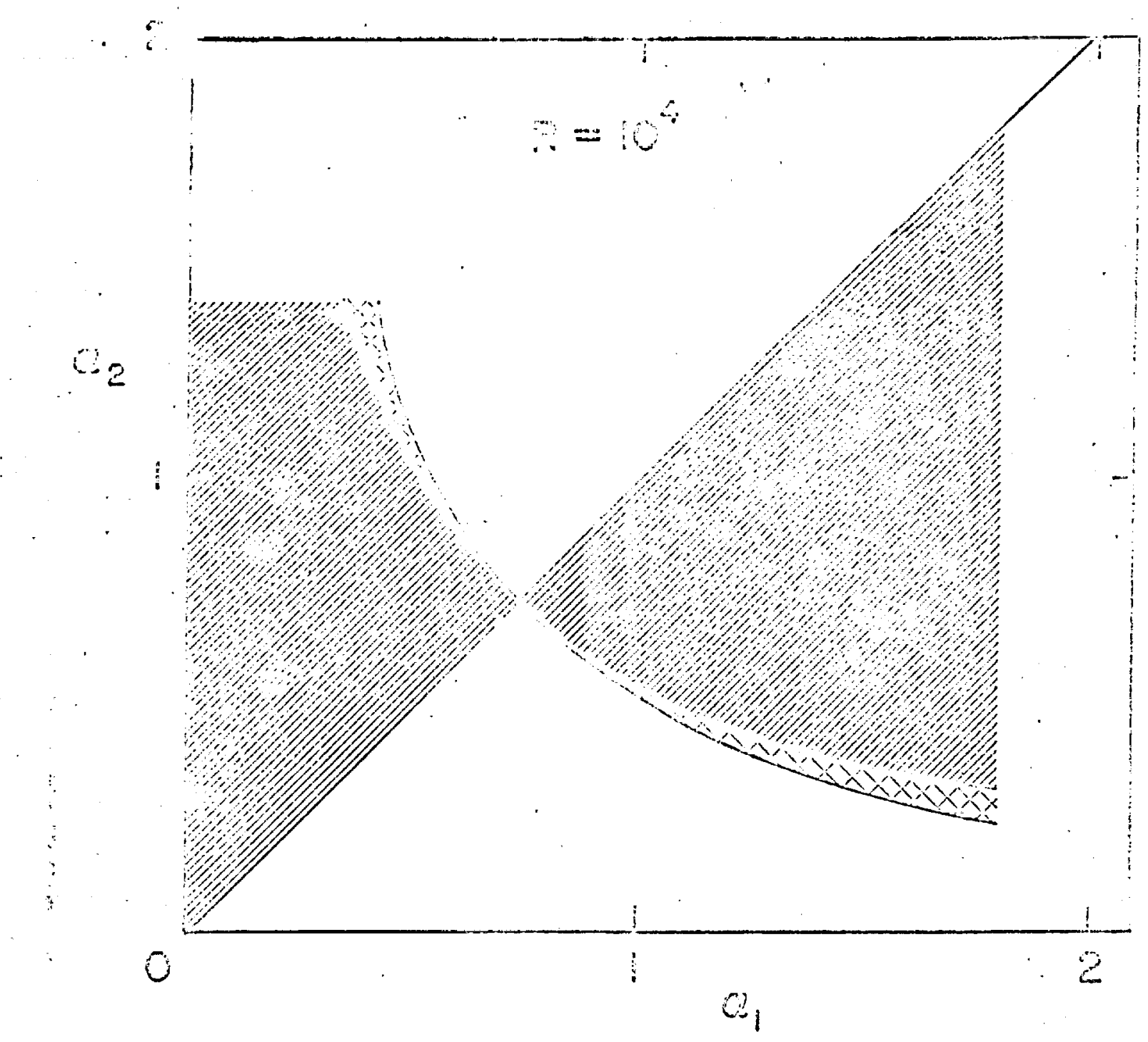

$1 \%$ 


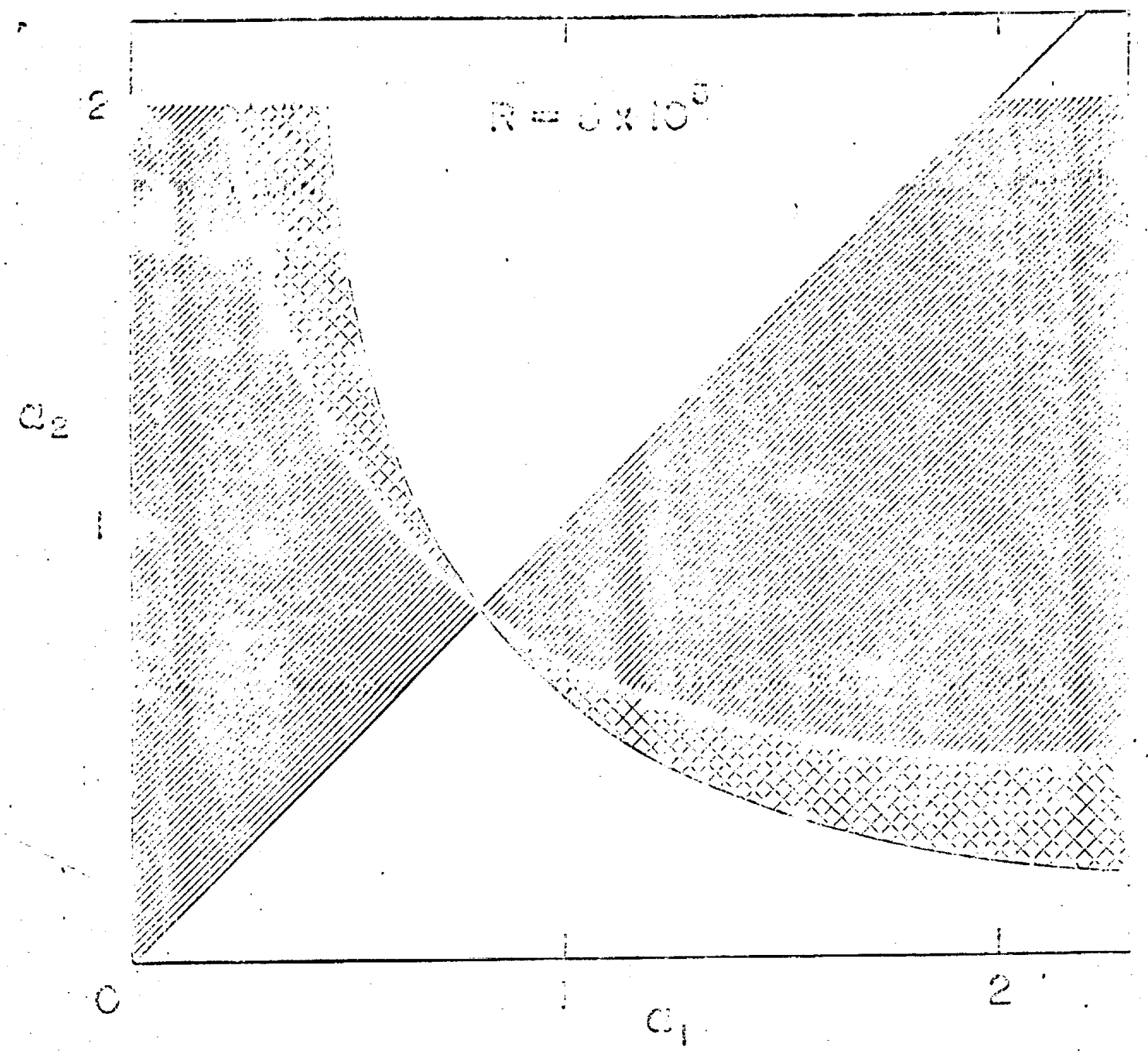

